\title{
Spike solutions for nonlinear Schrödinger equations in 2D with vanishing potentials
}

\author{
João Marcos do $\mathrm{O}^{1} \cdot$ Elisandra Gloss ${ }^{2} \cdot$ Federica Sani $^{3}$
}

Received: 22 August 2018 / Accepted: 9 April 2019 / Published online: 19 April 2019

(c) Fondazione Annali di Matematica Pura ed Applicata and Springer-Verlag GmbH Germany, part of Springer Nature 2019

\section{Abstract}

We consider $\varepsilon$-perturbed nonlinear Schrödinger equations of the form

$$
-\varepsilon^{2} \Delta u+V(x) u=Q(x) f(u) \text { in } \mathbb{R}^{2},
$$

where $V$ and $Q$ behave like $(1+|x|)^{-\alpha}$ with $\alpha \in(0,2)$ and $(1+|x|)^{-\beta}$ with $\beta \in$ $(\alpha,+\infty)$, respectively. When $f$ has subcritical exponential growth-by means of a weighted Trudinger-Moser-type inequality and the mountain pass theorem in weighted Sobolev spaces - we prove the existence of nontrivial mountain pass solutions, for any $\varepsilon>0$, and in the semi-classical limit, these solutions concentrate at a global minimum point of $\mathcal{A}=V / Q$. Our existence result holds also when $f$ has critical growth, for any $\varepsilon>0$.

Keywords Semi-classical limit $\cdot$ Schrödinger equations · Vanishing potentials · Weighted Trudinger-Moser inequality $\cdot$ Exponential growth

Mathematics Subject Classification $35 \mathrm{~J} 20 \cdot 35 \mathrm{Q} 55 \cdot 35 \mathrm{~B} 40 \cdot 35 \mathrm{~A} 23$

\section{Introduction and main results}

We deal with $\varepsilon$-perturbed nonlinear Schrödinger equations of the form

$$
-\varepsilon^{2} \Delta u+V(x) u=Q(x) f(u) \quad \text { in } \mathbb{R}^{2}
$$

Federica Sani

federica.sani@unimi.it

João Marcos do Ó

jmbo@pq.cnpq.br

Elisandra Gloss

elisandra@mat.ufpb.br

1 Universidade de Brasília, Brasília, DF 70910-900, Brazil

2 Universidade Federal da Paraíba, João Pessoa, PB 58051-900, Brazil

3 Università degli Studi di Milano, via Cesare Saldini 50, 20133 Milan, Italy 
where $\varepsilon>0$, and we mainly consider nonlinear terms $f=f(s)$ with subcritical exponential growth. We are interested in the case when the potential $V$ and the weight $Q$ are positive, bounded from above, and decay to zero at infinity. More precisely, we assume that for some $\alpha$ and $\beta$ in the range

$$
\alpha \in(0,2) \quad \text { and } \quad \beta \in(\alpha,+\infty)
$$

the following decay conditions hold

(V) $V \in \mathcal{C}^{1}\left(\mathbb{R}^{2}\right), V(x) \sim|x|^{-\alpha}$ as $|x| \rightarrow+\infty$, and

$$
\frac{a}{1+|x|^{\alpha}} \leq V(x) \leq A, \quad \text { where } a, A>0
$$

(Q) $Q \in \mathcal{C}^{1}\left(\mathbb{R}^{2}\right), Q(x) \sim|x|^{-\beta}$ as $|x| \rightarrow+\infty$, and

$$
0<Q(x) \leq \frac{b}{1+|x|^{\beta}}, \quad \text { where } b>0 .
$$

Inspired by [6], and complementing the result obtained in [21], we study the existence of mountain pass solutions for $\left(\mathcal{P}_{\varepsilon}\right)$, for any fixed $\varepsilon>0$, and their concentration behavior in the semi-classical limit, i.e., when $\varepsilon \rightarrow 0$.

In the case of subcritical polynomial nonlinearities in higher dimensions, with more general vanishing potentials $V$ and more general weights $Q$, concentration properties of solutions are extensively studied by means of suitable adaptations of the penalization method of del Pino and Felmer [17]. See, e.g., [10-12,27] and the references therein.

In the case of exponential nonlinearities in 2D, up to our knowledge, we have only the result of Fei and Yin [22], and a further generalization [15]. In [22] and [15], via penalization method, the authors construct solutions to $\left(\mathcal{P}_{\varepsilon}\right)$, with $\varepsilon>0$ sufficiently small, concentrating around a single point when

- $V$ can vanish at infinity, and $Q$ does not have to be bounded,

- $f(s)=|s|^{p-2} s \mathrm{e}^{\alpha_{0} s^{2}}$ with $p>2$ and $\alpha_{0}>0$.

Adapting the method of proof introduced by Ambrosetti, Felli and Malchiodi in [6], we analyze the concentration properties of mountain pass solutions to $\left(\mathcal{P}_{\varepsilon}\right)$ for a more restrictive class of weights and nonlinearities (indeed, we consider subcritical exponential nonlinearities), but what we gain is a more precise information about the limiting profile in the semi-classical limit, see Theorem 1.3. Moreover, differently from [22] and [15], our existence result holds without any restrictions on $\varepsilon>0$ in both the subcritical and critical case, see Theorems 1.2 and 1.4.

We point out that, in the case of exponential nonlinearities in $2 \mathrm{D}$, few results about the study of the semi-classical limit are available in the literature even for potentials $V$ which are positive and bounded away from zero. We refer the reader to $[4,5,9,13,20,25,36,38]$.

\subsection{Variational framework}

We apply the minimax variational method in a suitable weighted space. Let $p \geq 1$, and let us consider a weight function $w: \mathbb{R} \rightarrow[0,+\infty)$; we denote by $L_{w}^{p}\left(\mathbb{R}^{2}\right)$ the weighted $L^{p}$-space consisting of all measurable functions $u: \mathbb{R}^{2} \rightarrow \mathbb{R}$ satisfying

$$
\int_{\mathbb{R}^{2}} w(x)|u|^{p} \mathrm{~d} x<+\infty .
$$


Assuming $(V)$ with $\alpha \in(0,2]$, we introduce the weighted Sobolev space

$$
H_{V}^{1}\left(\mathbb{R}^{2}\right):=\left\{u \in L_{V}^{2}\left(\mathbb{R}^{2}\right):|\nabla u| \in L^{2}\left(\mathbb{R}^{2}\right)\right\},
$$

and for any fixed $\varepsilon>0$, we denote by $\mathcal{H}_{\varepsilon}$ the Hilbert space $H_{V}^{1}\left(\mathbb{R}^{2}\right)$ with inner product

$$
\langle u, v\rangle_{\varepsilon}:=\int_{\mathbb{R}^{2}}\left[\varepsilon^{2} \nabla u \cdot \nabla v+V(x) u v\right] \mathrm{d} x,
$$

and norm

$$
\|u\|_{\varepsilon}^{2}:=\langle u, u\rangle_{\varepsilon}=\varepsilon^{2}\|\nabla u\|_{2}^{2}+\int_{\mathbb{R}^{2}} V(x) u^{2} \mathrm{~d} x .
$$

The embedding results obtained in [32] (see also Theorem 2.5) ensure that if the weight $Q$ is of the form $\left(1+|x|^{\beta}\right)^{-1}$, then

$$
\mathcal{H}_{\varepsilon} \hookrightarrow L_{Q}^{p}\left(\mathbb{R}^{2}\right) \quad \forall p \in[2,+\infty)
$$

provided ( $Q$ ) holds with $\beta \in[\alpha,+\infty)$ (see [32, Example 20.6]). The above embeddings are compact if we restrict the range of $\beta$ and we assume $\beta>\alpha$.

It turns out that functions belonging to $\mathcal{H}_{\varepsilon}$ satisfy a weighted exponential integrability condition and the maximal growth allowed for a variational study of $\left(\mathcal{P}_{\varepsilon}\right)$ is determined by the following weighted Trudinger-Moser-type inequality in $\mathcal{H}_{\varepsilon}$.

Theorem 1.1 Suppose that $(V)$ and $(Q)$ hold with $\alpha \in(0,2)$ and $\beta \in[\alpha,+\infty)$, respectively. For any $\gamma>0$ and any $u \in H_{V}^{1}\left(\mathbb{R}^{2}\right)$, we have

$$
\int_{\mathbb{R}^{2}} Q(x)\left(\mathrm{e}^{\gamma u^{2}}-1\right) \mathrm{d} x<+\infty .
$$

Moreover, for any fixed $\varepsilon>0$,

$$
\sup _{u \in \mathcal{H}_{\varepsilon},\|u\|_{\varepsilon} \leq \varepsilon} \int_{\mathbb{R}^{2}} Q(x)\left(\mathrm{e}^{\gamma u^{2}}-1\right) \mathrm{d} x \begin{cases}<+\infty & \text { if } 0<\gamma<4 \pi, \\ =+\infty & \text { if } \gamma>4 \pi .\end{cases}
$$

The above theorem is proved in [21, Theorem 4.1] with $\alpha \in(0,2)$ and $\beta \in[2,+\infty)$, and hence, it improves [21, Theorem 4.1] with respect to the range of the parameter $\beta$.

In view of Theorem 1.1, we say that a nonlinear term $f$ has subcritical exponential growth at infinity if

$$
\lim _{|s| \rightarrow+\infty} \frac{|f(s)|}{\mathrm{e}^{\gamma s^{2}}}=0 \text { for any } \gamma>0
$$

while $f$ has critical exponential growth at infinity if

$$
\text { there exists } \gamma_{0}>0 \text { such that } \lim _{|s| \rightarrow+\infty} \frac{|f(s)|}{\mathrm{e}^{\gamma s^{2}}}= \begin{cases}0 & \text { if } \gamma>\gamma_{0}, \\ +\infty & \text { if } \gamma<\gamma_{0} .\end{cases}
$$

We recall that the above notions of subcritical and critical growth were first introduced in $[2,16]$ to study Dirichlet problems on bounded domains of $\mathbb{R}^{2}$. Clearly, in the case of bounded domains the notion of critical growth is governed by the classical Trudinger-Moser inequality [28]. 


\subsection{Subcritical case}

The following classical assumptions on the nonlinear term $f=f(s)$ are settled to guarantee a good mountain pass geometry:

$$
\begin{gathered}
\lim _{s \rightarrow 0} \frac{f(s)}{s}=0, \\
\exists \mu>2 \text { such that } 0<\mu F(s):=\mu \int_{0}^{s} f(t) \mathrm{d} t \leq s f(s) \quad \forall s \in \mathbb{R} \backslash\{0\} .
\end{gathered}
$$

Theorem 1.2 [Existence] Assume $(V)$ and $(Q)$ with $\alpha$ and $\beta$ in the range (1.1). Let $f: \mathbb{R} \rightarrow$ $\mathbb{R}$ be a continuous function, with subcritical exponential growth $\left(f_{0}\right)$, satisfying $\left(f_{1}\right)$ and $\left(f_{2}\right)$. Then equation $\left(\mathcal{P}_{\varepsilon}\right)$ has a positive mountain pass solution $u_{\varepsilon} \in \mathcal{H}_{\varepsilon}$, and $u_{\varepsilon} \in L^{2}\left(\mathbb{R}^{2}\right)$, for any $\varepsilon>0$. Moreover, there exists $C>0$ such that for any $\varepsilon>0$ sufficiently small we have

$$
\int_{\mathbb{R}^{2}} u_{\varepsilon}^{2} \mathrm{~d} x \leq C \varepsilon^{2} .
$$

The solution $u_{\varepsilon}$ found in Theorem 1.2 belongs to $\mathcal{H}_{\varepsilon}$, hence a priori it is not obvious that indeed $u_{\varepsilon} \in H^{1}\left(\mathbb{R}^{2}\right)$, and $u_{\varepsilon}$ is a weak solution of $\left(\mathcal{P}_{\varepsilon}\right)$ in the sense that

$$
\left\langle u_{\varepsilon}, v\right\rangle_{\varepsilon}-\int_{\mathbb{R}^{2}} Q(x) f\left(u_{\varepsilon}\right) v \mathrm{~d} x=0 \quad \forall v \in \mathcal{H}_{\varepsilon} .
$$

If in addition we assume that $f \in \mathcal{C}^{0, \sigma}(\mathbb{R})$ with $\sigma \in(0,1)$, then, by local elliptic regularity theory, $u_{\varepsilon} \in \mathcal{C}^{2}\left(\mathbb{R}^{2}\right)$ is a classical solution to $\left(\mathcal{P}_{\varepsilon}\right)$. In the semi-classical limit, i.e., when $\varepsilon \rightarrow 0$, we show that $u_{\varepsilon}$ are spike solutions concentrating at a global minimum point of the auxiliary potential $\mathcal{A}:=V / Q$. Clearly, $(V)$ and $(Q)$ with $\alpha$ and $\beta$ in the range (1.1) ensure that $\mathcal{A} \in \mathcal{C}\left(\mathbb{R}^{2}\right)$ is positive, $\lim _{|x| \rightarrow+\infty} \mathcal{A}(x)=+\infty$, and $\mathcal{A}$ has a positive global minimum.

Theorem 1.3 [Concentration] Assume $(V)$ and $(Q)$ with $\alpha$ and $\beta$ in the range (1.1), and assume that $f \in \mathcal{C}^{0, \sigma}(\mathbb{R})$ with $\sigma \in(0,1)$ satisfies $\left(f_{0}\right),\left(f_{1}\right)$ and $\left(f_{2}\right)$. The mountain pass solutions $u_{\varepsilon}$ found in Theorem 1.2 concentrate at a global minimum point $x^{*}$ of $\mathcal{A}$. More precisely, for any $\varepsilon>0$ sufficiently small, there exists a maximum point $x_{\varepsilon}$ of $u_{\varepsilon}$ such that $x_{\varepsilon} \rightarrow x^{*}$ as $\varepsilon \rightarrow 0$, and for any such $x_{\varepsilon}$,

$$
u_{\varepsilon}(x)=U^{*}\left(\frac{x-x_{\varepsilon}}{\varepsilon}\right)+\omega_{\varepsilon}(x) \text { as } \varepsilon \rightarrow 0,
$$

where the error term $\omega_{\varepsilon} \rightarrow 0$ in $\mathcal{C}_{\text {loc }}^{2}\left(\mathbb{R}^{2}\right)$ and in $L^{\infty}\left(\mathbb{R}^{2}\right)$, as $\varepsilon \rightarrow 0$, and $U^{*}$ is a ground-state solution of the limiting problem

$$
-\Delta U+V\left(x^{*}\right) U=Q\left(x^{*}\right) f(U) \text { in } \mathbb{R}^{2} .
$$

Moreover, the maximum point $x_{\varepsilon}$ is basically unique, in the sense that if $y_{\varepsilon}$ is a maximum point of $u_{\varepsilon}$ with $y_{\varepsilon} \neq x_{\varepsilon}$ then $\left|x_{\varepsilon}-y_{\varepsilon}\right| \leq C \varepsilon$. If in addition $f$ is increasing on $(0,+\infty)$, then $x_{\varepsilon}$ is indeed the unique local (and hence global) maximum point of $u_{\varepsilon}$.

\subsection{Critical case}

In order to apply the mountain pass theorem in the critical case, we require the following additional assumptions: 


$$
\begin{gathered}
\exists s_{0}, M_{0}>0 \text { such that } 0<F(s) \leq M_{0}|f(s)| \quad \forall|s| \geq s_{0}, \\
\lim _{|s| \rightarrow+\infty} \frac{s f(s)}{\mathrm{e}^{\gamma_{0} s^{2}}}=+\infty, \quad \text { with } \gamma_{0}>0 \text { given by } f_{0}^{\prime} .
\end{gathered}
$$

Note that, condition $\left(f_{3}\right)$ is used to prove the convergence of the nonlinear part of the energy functional along Palais-Smale sequences. This convergence ensures that the critical point obtained by means of the mountain pass theorem is in fact nontrivial. The growth condition $\left(f_{4}\right)$ is needed to guarantee that Palais-Smale sequences at the mountain pass level are convergent up to subsequences.

The following existence result is already proved in [21, Theorem 2.1, Proposition 2.2] with $\alpha \in(0,2)$ and $\beta \in[2,+\infty)$, our improvement concerns the range of the parameter $\beta$.

Theorem 1.4 [Existence] Assume $(V)$ and $(Q)$ with $\alpha$ and $\beta$ in the range (1.1). Let $f$ : $\mathbb{R} \rightarrow \mathbb{R}$ be a continuous function with critical exponential growth $\left(f_{0}^{\prime}\right)$ for some $\gamma_{0}>0$. If $f$ satisfies $\left(f_{1}\right),\left(f_{2}\right),\left(f_{3}\right)$ and $\left(f_{4}\right)$, then equation $\left(\mathcal{P}_{\varepsilon}\right)$ has a nontrivial mountain pass solution $u_{\varepsilon} \in \mathcal{H}_{\varepsilon}$, and $u_{\varepsilon} \in L^{2}\left(\mathbb{R}^{2}\right)$, for any $\varepsilon>0$.

For any fixed $\varepsilon>0$, the growth condition $\left(f_{4}\right)$ can be replaced by the weaker condition

$$
\liminf _{|s| \rightarrow+\infty} \frac{s f(s)}{\mathrm{e}^{\gamma_{0} s^{2}}}>\mathcal{M}_{\varepsilon}=\mathcal{M}_{\varepsilon}(V, Q):=\inf _{r>0} \frac{4 \varepsilon^{2} e^{\frac{1}{2 \varepsilon^{2}} r^{2} V_{\max , r}}}{\gamma_{0} r^{2} Q_{\min , r}}
$$

where

$$
V_{\max , r}:=\max _{|x| \leq r} V(x)>0 \quad \text { and } \quad Q_{\min , r}:=\min _{|x| \leq r} Q(x)>0 .
$$

Remark 1.5 Clearly, if $\alpha$ and $\beta$ are in the range (1.1) and we set $V(x)=a\left(1+|x|^{\alpha}\right)^{-1}$ and $Q(x)=b\left(1+|x|^{\beta}\right)^{-1}$, for some $a>0$ and $b>0$, then $\mathcal{M}_{\varepsilon} \in(0,+\infty)$ and $\lim _{\varepsilon \rightarrow 0} \mathcal{M}_{\varepsilon}=$ $+\infty$.

\subsection{Organization of the paper}

In Sect. 2, we prove the weighted Trudinger-Moser inequality stated in Theorem 1.1, and we pay particular attention to deduce some exponential integral uniform estimates for $\mathcal{H}_{\varepsilon}$ functions (see Lemma 2.6) which will be crucial to show the $L^{2}$-integrability of weak solutions to $\left(\mathcal{P}_{\varepsilon}\right)$, and to study the concentration behavior of mountain pass solutions to $\left(\mathcal{P}_{\varepsilon}\right)$.

Sections 3-5 are devoted to the case of nonlinearities with subcritical exponential growth. More precisely in Sect. 3, we prove the first part of Theorem 1.2, i.e., the existence result in the subcritical case. In Sect. 4 , we obtain the $L^{2}$-integrability of weak solutions to $\left(\mathcal{P}_{\varepsilon}\right)$ (see Proposition 4.4), and the $L^{2}$-uniform bound for weak solutions described by Proposition 4.3, which holds for mountain pass solutions to $\left(\mathcal{P}_{\varepsilon}\right)$ in view of Corollary 5.4. Hence, the results of Sect. 4 complete the proof of Theorem 1.2.

Also in Sect. 4, we pay special attention to integral uniform estimates for weak solutions to $\left(\mathcal{P}_{\varepsilon}\right)$, and in particular, Lemma 4.2 will be crucial to analyze the concentration behavior of mountain pass solutions to $\left(\mathcal{P}_{\varepsilon}\right)$. This analysis will be developed in Sect. 5 .

Finally, Sect. 6 is devoted to the proof of Theorem 1.4, i.e., the existence result in the critical case. 


\subsection{Open problems}

Both the case $\alpha=2$ with $\beta \in[2,+\infty)$ and the case $\gamma=4 \pi$ are left open by Theorem 1.1. Does a weighted Trudinger-Moser inequality hold in these cases?

The study of the concentration behavior of the mountain pass solutions found in Theorem 1.4 , in the critical exponential case, is still open. Following our argument, it is not clear how to extend the validity of Lemma 4.2 to the critical case.

\subsection{Notations}

We denote by $B(x, R) \subset \mathbb{R}^{2}$ the closed ball of radius $R>0$ centered at $x \in \mathbb{R}^{2}$ and, to simplify notations, we set $B_{R}:=B(0, R)$ and $B_{R}^{\mathrm{c}}:=\mathbb{R}^{2} \backslash B_{R}$.

\section{A weighted Trudinger-Moser-type inequality and integral uniform estimates for $\mathcal{H}_{\varepsilon}$-functions}

This section is mainly devoted to the proof of the weighted Trudinger-Moser inequality expressed by Theorem 1.1. As a by-product of the proof of Theorem 1.1, we will deduce again the embedding results obtained in [32], see Theorem 2.5. Finally, having in mind the study of the concentration behavior of solutions to $\left(\mathcal{P}_{\varepsilon}\right)$, we complete this section with some integral estimates for functions belonging to $\mathcal{H}_{\varepsilon}$ which are uniform with respect to $\varepsilon>0$, see Lemma 2.6.

As already mentioned, Theorem 1.1 improves [21, Theorem 1.1] with respect to the range of the parameter $\beta$ which determines the decay of the weight $Q$ at infinity. More precisely, the arguments of the proof of [21, Theorem 1.1] enable to deduce the validity of (1.3)

$$
\text { for any } \alpha \text { and } \beta \text { in the range } \alpha \in(0,2) \text { and } \beta \in[2,+\infty) \text {, }
$$

while here we will show that (1.3) actually holds

$$
\text { for any } \alpha \text { and } \beta \text { in the range } \alpha \in(0,2) \text { and } \beta \in[\alpha,+\infty) \text {. }
$$

This improvement can be reached by following closely the method adopted in [21], and for this reason, we will only give the details of the proof of some estimates with explicit dependence on $\varepsilon$ that will be crucial to obtain the concentration result for equation $\left(\mathcal{P}_{\varepsilon}\right)$.

The main difference with respect to the proof of [21, Theorem 1.1] can be summarized as follows. The method adopted in [21] combines ideas of Kufner and Opic [32] with a local version of the classical Trudinger-Moser inequality pointed out by Yang and Zhu in [37] (see also [21, Lemma 4.3]). Instead of applying that local Trudinger-Moser inequality, we will use locally the following well known global result.

Theorem 2.1 ([1,18,30,31,33]) There exist constants $C_{\gamma}>0$ such that

$$
\sup _{u \in H^{1}\left(\mathbb{R}^{2}\right),\|\nabla u\|_{2} \leq 1} \frac{1}{\|u\|_{2}^{2}} \int_{\mathbb{R}^{2}}\left(\mathrm{e}^{\gamma u^{2}}-1\right) \mathrm{d} x \begin{cases}\leq C_{\gamma} & \text { if } 0<\gamma<4 \pi, \\ =+\infty & \text { if } \gamma \geq 4 \pi .\end{cases}
$$

First, we derive an integral estimate in the exterior of a large ball which is uniform with respect $\varepsilon$, provided $\varepsilon>0$ is sufficiently small.

Proposition 2.2 Suppose that $(V)$ and $(Q)$ hold with $\alpha \in(0,2)$ and $\beta \in[\alpha,+\infty)$, respectively, and let $0<\gamma<4 \pi$. 
(I) There exists $\tilde{n}=\tilde{n}(\varepsilon, \gamma, a, \alpha)>1$ such that for any $n \geq \tilde{n}$ we have

$$
\int_{B_{3 n}^{c}} Q(x)\left(\mathrm{e}^{\gamma u^{2}}-1\right) \mathrm{d} x \leq C_{\tilde{n}, a, \alpha, b, \beta} \int_{B_{n}^{c}} V(x) u^{2} \mathrm{~d} x \quad \forall u \in \mathcal{H}_{\varepsilon} \text { with }\|u\|_{\varepsilon} \leq \varepsilon .
$$

(II) If in addition $\beta>\alpha$, then for any $\eta>0$ there exists $\tilde{n}=\tilde{n}(\varepsilon, \eta, \gamma, a, \alpha)>1$ such that for any $n \geq \tilde{n}$ we have

$$
\int_{B_{3 n}^{c}} Q(x)\left(\mathrm{e}^{\gamma u^{2}}-1\right) \mathrm{d} x \leq \eta \int_{B_{n}^{c}} V(x) u^{2} \mathrm{~d} x \quad \forall u \in \mathcal{H}_{\varepsilon} \text { with }\|u\|_{\varepsilon} \leq \varepsilon .
$$

In both cases, if $\varepsilon \in(0,1]$ then $\tilde{n}$ can be chosen independently of $\varepsilon$.

Proof For any fixed $n \geq \tilde{n}$, with $\tilde{n}>>1$ to be chosen during the proof, we consider the covering of $B_{n}^{\mathrm{c}}$ consisting of annuli of the form

$$
A_{n}^{\sigma}:=\left\{x \in B_{n}^{\mathrm{c}}|| x \mid<\sigma\right\}=\left\{x \in \mathbb{R}^{2}|n<| x \mid<\sigma\right\} \text {, with } \sigma>n .
$$

As in [21, Section 4.2], we apply the Besicovitch covering lemma (see [21, Lemma 4.5] and $[21,(4.8)])$ which gives the existence of a sequence of points $\left\{x_{k}\right\}_{k} \in A_{\tilde{n}}^{\sigma}$ and the existence of a universal constant $\theta>0$ such that

$$
A_{\tilde{n}}^{\sigma} \subseteq \bigcup_{k} U_{k}^{1 / 2}, \quad \text { where } U_{k}^{1 / 2}:=B\left(x_{k}, \frac{1}{2} \frac{\left|x_{k}\right|}{3}\right),
$$

and

$$
\sum_{k} \chi_{U_{k}}(x) \leq \theta \text { for any } x \in \mathbb{R}^{2},
$$

where $\chi_{U_{k}}$ is the characteristic function of $U_{k}:=B\left(x_{k},\left|x_{k}\right| / 3\right)$.

Let $u \in \mathcal{H}_{\varepsilon}$ be such that $\|u\|_{\varepsilon} \leq \varepsilon$. We begin with the estimate of the weighted exponential integral of $u$ on $A_{3 n}^{\sigma}$ with $n \geq \tilde{n}$ and $\sigma>3 n$. Clearly,

$$
A_{3 n}^{\sigma} \subset A_{\tilde{n}}^{\sigma} \subseteq \bigcup_{k} U_{k}^{1 / 2},
$$

and if we consider the set of indices $K_{n, \sigma}:=\left\{k \in \mathbb{N} \mid U_{k}^{1 / 2} \cap B_{3 n}^{\mathrm{c}} \neq \varnothing\right\}$ then we have

$$
A_{3 n}^{\sigma} \subseteq \bigcup_{k \in K_{n, \sigma}} U_{k}^{1 / 2}
$$

Therefore, we get

$$
\int_{A_{3 n}^{\sigma}} Q(x)\left(\mathrm{e}^{\gamma u^{2}}-1\right) \mathrm{d} x \leq \sum_{k \in K_{n, \sigma}} \int_{U_{k}^{1 / 2}} Q(x)\left(\mathrm{e}^{\gamma u^{2}}-1\right) \mathrm{d} x .
$$

In order to estimate the single terms of the series on the right-hand side, few remarks about the choice of the balls $U_{k}^{1 / 2}$ and $U_{k}$ are needed. Since

$$
2\left|x_{k}\right| / 3 \leq|y| \leq 4\left|x_{k}\right| / 3 \quad \forall y \in U_{k},
$$

we have the existence of $C_{\alpha}, C_{\beta}>0$ such that

$$
V(y) \geq \frac{a}{1+|y|^{\alpha}} \geq \frac{a}{1+C_{\alpha}\left|x_{k}\right|^{\alpha}} \quad \forall y \in U_{k},
$$


and

$$
Q(y) \leq \frac{b}{1+|y|^{\beta}} \leq \frac{b}{1+C_{\beta}\left|x_{k}\right|^{\beta}} \quad \forall y \in U_{k} .
$$

Furthermore, it is easy to see that if $U_{k} \cap B_{3 n}^{\mathrm{c}} \neq \varnothing$ then $U_{k} \subset B_{n}^{\mathrm{c}}$, and this yields

$$
\bigcup_{k \in K_{n, \sigma}} U_{k}^{1 / 2} \subseteq \bigcup_{k \in K_{n, \sigma}} U_{k} \subseteq B_{n}^{\mathrm{c}} \subseteq B_{\tilde{n}}^{\mathrm{c}}
$$

Let us fix $k \in K_{n, \sigma}$. We have

$$
\int_{U_{k}^{1 / 2}} Q(x)\left(\mathrm{e}^{\gamma u^{2}}-1\right) \mathrm{d} x \leq \frac{b}{1+C_{\beta}\left|x_{k}\right|^{\beta}} \int_{U_{k}^{1 / 2}}\left(\mathrm{e}^{\gamma u^{2}}-1\right) \mathrm{d} x,
$$

and we will apply Theorem 2.1 to estimate the unweighted integral on $U_{k}^{1 / 2}$. To this aim, we introduce a cutoff function $\varphi_{k} \in \mathcal{C}_{0}^{\infty}\left(U_{k}\right)$ satisfying

$$
0 \leq \varphi_{k} \leq 1 \text { in } U_{k}, \quad \varphi_{k} \equiv 1 \text { in } U_{k}^{1 / 2}, \quad \text { and } \quad|\nabla \varphi| \leq C /\left|x_{k}\right| \text { in } U_{k},
$$

for some universal constant $C>0$. Then $\varphi_{k} u \in H_{0}^{1}\left(U_{k}\right) \subset H^{1}\left(\mathbb{R}^{2}\right)$ and we may estimate

$$
\begin{aligned}
\int_{U_{k}}\left|\nabla\left(\varphi_{k} u\right)\right|^{2} \mathrm{~d} x & \leq(1+\delta) \int_{U_{k}}|\nabla u|^{2} \mathrm{~d} x+\left(1+\frac{1}{\delta}\right) \frac{C^{2}}{\left|x_{k}\right|^{2}} \int_{U_{k}} u^{2} \mathrm{~d} x \\
& \leq(1+\delta) \int_{U_{k}}|\nabla u|^{2} \mathrm{~d} x+\left(1+\frac{1}{\delta}\right) \frac{C^{2}}{a} \frac{1+C_{\alpha}\left|x_{k}\right|^{\alpha}}{\left|x_{k}\right|^{2}} \int_{U_{k}} V(x) u^{2} \mathrm{~d} x,
\end{aligned}
$$

where we also used (2.3). The above estimate holds for any $\delta>0$; by assumption $\gamma \in(0,4 \pi)$, and we can choose $\delta \in(0,1)$ so that

$$
\gamma /(1-\delta)<4 \pi
$$

Recalling that $k \in K_{n, \sigma}$, in view of (2.4), we have that $x_{k} \in B_{\tilde{n}}^{\mathrm{c}}$. Since $\alpha \in(0,2)$, there exists $\tilde{n}=\tilde{n}(\varepsilon, \delta, a, \alpha)$ sufficiently large such that

$$
\left(1+\frac{1}{\delta}\right) \frac{C^{2}}{a} \frac{1+C_{\alpha}\left|x_{k}\right|^{\alpha}}{\left|x_{k}\right|^{2}} \leq \frac{1+\delta}{\varepsilon^{2}} \quad \forall k \in K_{n, \sigma}, n \geq \tilde{n},
$$

and we can take $\tilde{n}=\tilde{n}(1, \delta, a, \alpha)$ for any $\varepsilon \in(0,1]$. In this way, we get

$$
\int_{U_{k}}\left|\nabla\left(\varphi_{k} u\right)\right|^{2} \mathrm{~d} x \leq \frac{(1+\delta)}{\varepsilon^{2}} \int_{U_{k}}\left(\varepsilon^{2}|\nabla u|^{2}+V(x) u^{2}\right) \mathrm{d} x \leq(1+\delta) .
$$

If we set $v_{k}:=\sqrt{1-\delta} \varphi_{k} u \in H^{1}\left(\mathbb{R}^{2}\right)$, then $\left\|\nabla v_{k}\right\|_{2}^{2} \leq 1-\delta^{2} \leq 1$. In view of (2.6), we can apply Theorem 2.1 to $v_{k}$ obtaining

$$
\int_{U_{k}^{1 / 2}}\left(\mathrm{e}^{\gamma u^{2}}-1\right) \mathrm{d} x=\int_{U_{k}^{1 / 2}}\left(\mathrm{e}^{\gamma\left(\varphi_{k} u\right)^{2}}-1\right) \mathrm{d} x \leq \int_{\mathbb{R}^{2}}\left(\mathrm{e}^{[\gamma /(1-\delta)] v_{k}^{2}}-1\right) \mathrm{d} x \leq C \int_{\mathbb{R}^{2}}\left|v_{k}\right|^{2} \mathrm{~d} x,
$$

for a positive constant $C=C(\gamma, \delta)$. Then we deduce

$$
\int_{U_{k}^{1 / 2}}\left(\mathrm{e}^{\gamma u^{2}}-1\right) \mathrm{d} x \leq C(1-\delta) \int_{U_{k}} u^{2} \mathrm{~d} x \leq C(1-\delta) \frac{1+C_{\alpha}\left|x_{k}\right|^{\alpha}}{a} \int_{U_{k}} V(x) u^{2} \mathrm{~d} x .
$$


Combining (2.2), (2.5) and (2.7), we get

$$
\begin{aligned}
\int_{A_{3 n}^{\sigma}} Q(x)\left(\mathrm{e}^{\gamma u^{2}}-1\right) \mathrm{d} x & \leq \frac{b}{a} C(1-\delta) \sum_{k \in K_{n, \sigma}} \frac{1+C_{\alpha}\left|x_{k}\right|^{\alpha}}{1+C_{\beta}\left|x_{k}\right|^{\beta}} \int_{U_{k}} V(x) u^{2} \mathrm{~d} x \\
& \leq \frac{b}{a} C(1-\delta) \sum_{k \in K_{n, \sigma}} \frac{1+C_{\alpha}\left|x_{k}\right|^{\alpha}}{1+C_{\beta}\left|x_{k}\right|^{\beta}} \int_{B_{n}^{\mathrm{c}}} V(x) u^{2} \chi_{U_{k}}(x) \mathrm{d} x
\end{aligned}
$$

where the last inequality follows from (2.4). Using again (2.4), we have

$$
\frac{1+C_{\alpha}\left|x_{k}\right|^{\alpha}}{1+C_{\beta}\left|x_{k}\right|^{\beta}} \leq \mathcal{B}_{n}:=\sup _{x \in B_{n}^{\mathrm{c}}} \frac{1+C_{\alpha}|x|^{\alpha}}{1+C_{\beta}|x|^{\beta}} \quad \forall k \in K_{n, \sigma} .
$$

Hence

$$
\int_{A_{3 n}^{\sigma}} Q(x)\left(\mathrm{e}^{\gamma u^{2}}-1\right) \mathrm{d} x \leq \frac{b}{a} C(1-\delta) \mathcal{B}_{n} \sum_{k \in K_{n, \sigma}} \int_{B_{n}^{\mathrm{c}}} V(x) u^{2} \chi_{U_{k}}(x) \mathrm{d} x,
$$

and, in view of (2.1),

$$
\int_{A_{3 n}^{\sigma}} Q(x)\left(\mathrm{e}^{\gamma u^{2}}-1\right) \mathrm{d} x \leq \frac{b}{a} C(1-\delta) \theta \mathcal{B}_{n} \int_{B_{n}^{\mathrm{c}}} V(x) u^{2} \mathrm{~d} x .
$$

Letting $\sigma \rightarrow+\infty$, we deduce the existence of $\tilde{n}=\tilde{n}(\varepsilon, \delta, a, \alpha)>>1$ such that for any $n \geq \tilde{n}$ we have

$$
\int_{B_{3 n}^{\mathrm{c}}} Q(x)\left(\mathrm{e}^{\gamma u^{2}}-1\right) \mathrm{d} x \leq b C \theta \mathcal{B}_{n} \int_{B_{n}^{\mathrm{c}}} V(x) u^{2} \mathrm{~d} x .
$$

Note that

$$
\lim _{n \rightarrow+\infty} \mathcal{B}_{n}=\lim _{n \rightarrow+\infty} \frac{1+C_{\alpha} n^{\alpha}}{1+C_{\beta} n^{\beta}}= \begin{cases}0 & \text { if } \beta>\alpha, \\ C_{\alpha} / C_{\beta} & \text { if } \beta=\alpha,\end{cases}
$$

and in conclusion (2.8) yields both (I) and (II).

In view of Proposition 2.2(I), the sharp inequality (1.3) follows by a simple adaptation of the arguments in [21]. More precisely, arguing as in [21], we can prove

Proposition 2.3 Suppose that $(V)$ and $(Q)$ hold with $\alpha \in(0,2)$ and $\beta \in[\alpha,+\infty)$, respectively.

(I) Let $0<\gamma<4 \pi$. There exists $\tilde{R}=\tilde{R}(\varepsilon, \gamma, a, \alpha)>0$ such that for any $R \geq \tilde{R}$ we have

$$
\int_{B_{R}} Q(x)\left(\mathrm{e}^{\gamma u^{2}}-1\right) \mathrm{d} x \leq C R^{2} \quad \forall u \in \mathcal{H}_{\varepsilon} \text { with }\|u\|_{\varepsilon} \leq \varepsilon,
$$

where $C>0$ is a universal constant. In particular, if $\varepsilon \in(0,1]$ then $\tilde{R}$ can be chosen independently of $\varepsilon$.

(II) For any $\gamma>4 \pi$ we have

$$
\sup _{u \in \mathcal{H}_{\varepsilon},\|u\|_{\varepsilon} \leq \varepsilon} \int_{\mathbb{R}^{2}} Q(x)\left(\mathrm{e}^{\gamma u^{2}}-1\right) \mathrm{d} x=+\infty .
$$


If $\varepsilon=1, \alpha \in(0,2)$ and $\beta \in[2,+\infty)$, Proposition 2.3(I) is nothing but the result obtained in [21, Section 4.1]. Since the argument of the proof in [21] is independent of $\beta$, it can be easily adapted to cover the case $\beta \in[\alpha,+\infty)$ with obvious modifications taking into account the presence of the parameter $\varepsilon>0$.

For the proof of the sharpness statement expressed by Proposition 2.3(II), we refer to the beginning of [21, Section 4]. We only point out the key point, that is: since $V$ and $Q$ are bounded away from zero by positive constants on compact subsets of $\mathbb{R}^{2}$, and $H^{1}\left(\mathbb{R}^{2}\right) \hookrightarrow \mathcal{H}_{\varepsilon}$, the sharpness statement is a direct consequence of the sharpness of the Trudinger-Moser inequality in $H^{1}\left(\mathbb{R}^{2}\right)$ due to Ruf [35] (see also [3] and [14, Remark 6.1]).

Since the space $\mathcal{C}_{0}^{\infty}\left(\mathbb{R}^{2}\right)$ is dense in $\mathcal{H}_{\varepsilon}$ (see [21, Section 3]), the validity of (1.2) can be derived from (1.3) using standard density arguments (see for instance [21, Section 4.3]).

With these remarks, the proof of Theorem 1.1 is completed.

Remark 2.4 For any fixed $\gamma \in(0,4 \pi)$, the family of suprema $\left\{S_{\gamma, \varepsilon}\right\}_{\varepsilon \in(0,1]}$, with $S_{\gamma, \varepsilon}$ defined by

$$
S_{\gamma, \varepsilon}:=\sup _{u \in \mathcal{H}_{\varepsilon},\|u\|_{\varepsilon} \leq \varepsilon} \int_{\mathbb{R}^{2}} Q(x)\left(\mathrm{e}^{\gamma u^{2}}-1\right) \mathrm{d} x,
$$

is bounded. In other words, for any $\gamma \in(0,4 \pi)$ there exists $C_{\gamma}>0$ such that

$$
S_{\gamma, \varepsilon} \leq C_{\gamma} \quad \text { for all } \varepsilon \in(0,1] \text {. }
$$

This is a consequence of the fact that both $\tilde{n}$ in Proposition 2.2(I) and $\tilde{R}$ in Proposition 2.3(I) can be chosen independently of $\varepsilon \in(0,1]$.

As a by-product of the proof of Proposition 2.2, we re-obtain the embedding results of Kufner and Opic [32]. More precisely,

Theorem 2.5 [32] If $(V)$ and $(Q)$ hold with $\alpha \in(0,2)$ and $\beta \in[\alpha,+\infty)$, respectively, then

$$
\mathcal{H}_{\varepsilon} \hookrightarrow L_{Q}^{p}\left(\mathbb{R}^{2}\right) \quad \forall p \in[2,+\infty),
$$

and there exists $C_{\varepsilon, p}>0$ such that

$$
\int_{\mathbb{R}^{2}} Q(x)|u|^{p} \mathrm{~d} x \leq C_{\varepsilon, p}\|u\|_{\varepsilon}^{2} \quad \forall u \in \mathcal{H}_{\varepsilon} .
$$

Moreover, if $\beta>\alpha$, then the above embeddings are compact.

Proof Since the spaces $\mathcal{H}_{\varepsilon}:=\left(H_{V}^{1}\left(\mathbb{R}^{2}\right),\|\cdot\|_{\varepsilon}\right)$ are isomorphic for all $\varepsilon>0$, without loss of generality we take $\varepsilon=1$. On the one hand $H_{V}^{1}\left(\mathbb{R}^{2}\right) \hookrightarrow H_{\text {loc }}^{1}\left(\mathbb{R}^{2}\right)$. On the other hand, for any $p \geq 2$ there exists $C_{p}>0$ such that $|t|^{p} \leq C_{p}\left(e^{t^{2}}-1\right)$ for any $t \in \mathbb{R}$, and hence (2.8) yields

$$
\lim _{n \rightarrow \infty} \sup _{u \in H_{V}^{1}\left(\mathbb{R}^{2}\right),\|u\| \leq 1} \int_{B_{n}^{\mathrm{c}}} Q(x)|u|^{p} \mathrm{~d} x=\left\{\begin{array}{cc}
0 & \text { if } \beta>\alpha \\
<+\infty & \text { if } \beta=\alpha .
\end{array}\right.
$$

Therefore, we can apply [32, Theorems 17.6 and 17.10] to deduce the continuity of the embeddings (2.9), and their compactness when $\beta>\alpha$.

The inequality expressed by Proposition 2.2(II) can be seen as a counterpart of [6, Proposition 7] for the 2D case. Since the integrals on the two side of the inequality are taken on 
different domains, following [6, Proposition 11], we show that the dependence on the same domain can be restored, and at once, we restate the result to obtain a more suitable tool for the study of concentration of solutions to $\left(\mathcal{P}_{\varepsilon}\right)$.

Lemma 2.6 Suppose that $(V)$ and $(Q)$ hold with $\alpha \in(0,2)$ and $\beta \in(\alpha,+\infty)$, respectively, i.e., with $\alpha$ and $\beta$ in the range (1.1). Let $M>0$, and let us consider any function $u \in \mathcal{H}_{\varepsilon}$ satisfying

$$
\|u\|_{\varepsilon}^{2} \leq M \varepsilon^{2} \quad \text { for some } \varepsilon>0 .
$$

Then for any $\gamma \in(0, \bar{\gamma})$, with $\bar{\gamma}=\bar{\gamma}(M)>0$, and for any $\eta>0$ there exists $\bar{R}>1$ such that

$$
\int_{B_{R}^{c}} Q(x)\left(\mathrm{e}^{\gamma u^{2}}-1\right) \mathrm{d} x \leq \eta \int_{B_{R}^{c}}\left(\varepsilon^{2}|\nabla u|^{2}+V(x) u^{2}\right) \mathrm{d} x \quad \forall R \geq \bar{R} .
$$

In particular, if $\varepsilon \in(0,1]$ then $\bar{R}$ can be chosen independently of $\varepsilon$.

Proof Let $R>1$. We consider a smooth non-decreasing function $\psi_{R, \varepsilon}: \mathbb{R}^{+} \rightarrow[0,1]$ satisfying

$$
\begin{gathered}
\psi_{R, \varepsilon}(r)=0 \text { if } 0 \leq r \leq R-\varepsilon R^{\alpha / 2}, \quad \psi_{R, \varepsilon}(r)=1 \text { if } r \geq R, \quad \text { and } \\
\left|\psi_{R, \varepsilon}^{\prime}(r)\right| \leq 2 /\left(\varepsilon R^{\alpha / 2}\right) \forall r \in \mathbb{R}^{+} .
\end{gathered}
$$

In polar coordinates $(r, \theta) \in[0,+\infty) \times \mathbb{S}^{1}$, we define

$$
u_{R, \varepsilon}(r, \theta):= \begin{cases}0 & \text { if } 0 \leq r \leq R-\varepsilon R^{\alpha / 2} \\ \psi_{R, \varepsilon}(r) u(2 R-r, \theta) & \text { if } R-\varepsilon R^{\alpha / 2} \leq r \leq R \\ u(r, \theta) & \text { if } r \geq R .\end{cases}
$$

On the annulus

$$
A_{R, \varepsilon}:=\left\{x \in \mathbb{R}^{2}\left|R-\varepsilon R^{\alpha / 2} \leq\right| x \mid \leq R\right\},
$$

arguing as in [6, Proposition 11], we get the estimate

$$
\int_{A_{R, \varepsilon}}\left(\varepsilon^{2}\left|\nabla u_{R, \varepsilon}\right|^{2}+V(x) u_{R, \varepsilon}^{2}\right) \mathrm{d} x \leq C \int_{B_{R}^{\mathrm{c}}}\left(\varepsilon^{2}|\nabla u|^{2}+V(x) u^{2}\right) \mathrm{d} x,
$$

where $C>0$ does not depend neither on $\varepsilon>0$ nor on $R>1$. Since $u_{R, \varepsilon} \equiv 0$ when $|x| \leq R-\varepsilon R^{\alpha / 2}$ and $u_{R, \varepsilon} \equiv u$ when $|x| \geq R$, we deduce

$$
\begin{aligned}
\left\|u_{R, \varepsilon}\right\|_{\varepsilon}^{2} & =\int_{B_{R-\varepsilon R^{\alpha} / 2}^{\mathrm{c}}}\left(\varepsilon^{2}\left|\nabla u_{R, \varepsilon}\right|^{2}+V(x) u_{R, \varepsilon}^{2}\right) \mathrm{d} x \leq(1+C) \int_{B_{R}^{\mathrm{c}}}\left(\varepsilon^{2}|\nabla u|^{2}+V(x) u^{2}\right) \mathrm{d} x \\
& \leq(1+C) M \varepsilon^{2},
\end{aligned}
$$

where we also used (2.10).

Next, the idea is to apply Proposition 2.2(II) to the auxiliary function

$$
w_{R, \varepsilon}:=u_{R, \varepsilon} / \sqrt{(1+C) M} .
$$

For any $\gamma>0$, we can estimate

$$
\begin{aligned}
\int_{B_{R}^{\mathrm{c}}} Q(x)\left(\mathrm{e}^{\gamma u^{2}}-1\right) \mathrm{d} x & =\int_{B_{R}^{\mathrm{c}}} Q(x)\left(\mathrm{e}^{\gamma u_{R, \varepsilon}^{2}}-1\right) \mathrm{d} x \leq \int_{B_{R-\varepsilon R^{\alpha} / 2}^{\mathrm{c}}} Q(x)\left(\mathrm{e}^{\gamma u_{R, \varepsilon}^{2}}-1\right) \mathrm{d} x \\
& =\int_{B_{R-\varepsilon R^{\alpha / 2}}^{\mathrm{c}}} Q(x)\left(\mathrm{e}^{\left.\gamma(1+C) M w_{R, \varepsilon}^{2}-1\right) \mathrm{d} x .}\right.
\end{aligned}
$$


If $\eta>0$ is arbitrarily fixed and $\tilde{n}=\tilde{n}(\varepsilon, \eta M, \gamma, a, \alpha)$ is given by Proposition 2.2(II), then, by choosing $\bar{R}>(\varepsilon \alpha / 2)^{2 /(2-\alpha)}$ such that $\bar{R}-\varepsilon \bar{R}^{\alpha / 2} \geq 3 \tilde{n}$, we have for any $R \geq \bar{R}$

$$
\begin{aligned}
\int_{B_{R}^{\mathrm{c}}} Q(x)\left(\mathrm{e}^{\gamma u^{2}}-1\right) \mathrm{d} x & \leq \int_{B_{R-\varepsilon R^{\alpha} / 2}^{\mathrm{c}}} Q(x)\left(\mathrm{e}^{\gamma(1+C) M w_{R, \varepsilon}^{2}}-1\right) \mathrm{d} x \\
& \leq(M \eta) \int_{\mathbb{R}^{2}} V(x) w_{R, \varepsilon}^{2} \mathrm{~d} x \leq \eta \int_{B_{R}^{\mathrm{c}}}\left(\varepsilon^{2}|\nabla u|^{2}+V(x) u^{2}\right) \mathrm{d} x,
\end{aligned}
$$

provided $0<\gamma<\bar{\gamma}$ with

$$
\bar{\gamma}:=4 \pi /[(1+C) M] .
$$

Clearly, Proposition 2.2(II) also guarantees that $\tilde{n}$, and hence $\bar{R}$, can be chosen independently of $\varepsilon \in(0,1]$.

The useful aspect of Lemma 2.6 is that neither the exponent $\bar{\gamma}>0$ nor the radius $\bar{R}>1$ depend on $\varepsilon \in(0,1]$.

\section{Subcritical case-existence}

In this section, we prove the existence of a mountain pass solution to $\left(\mathcal{P}_{\varepsilon}\right)$, for any $\varepsilon>0$, under the assumptions:

- $(V)$ and $(Q)$ hold with $\alpha$ and $\beta$ in the range (1.1),

- $f: \mathbb{R} \rightarrow \mathbb{R}$ is a continuous function, with subcritical exponential growth $\left(f_{0}\right)$, satisfying $\left(f_{1}\right)$ and $\left(f_{2}\right)$.

In other words, we prove the existence statement of Theorem 1.2, while the $L^{2}$-integrability of mountain pass solutions will be established in Proposition 4.4.

The functional associated with a variational approach to $\left(\mathcal{P}_{\varepsilon}\right)$ is defined as

$$
I_{\varepsilon}(u):=\frac{1}{2}\|u\|_{\varepsilon}^{2}-\int_{\mathbb{R}^{2}} Q(x) F(u) \mathrm{d} x \quad \forall u \in \mathcal{H}_{\varepsilon} .
$$

Moreover, $I_{\varepsilon} \in \mathcal{C}^{1}\left(\mathcal{H}_{\varepsilon}, \mathbb{R}\right)$ with

$$
I_{\varepsilon}^{\prime}[u](v):=\langle u, v\rangle_{\varepsilon}-\int_{\mathbb{R}^{2}} Q(x) f(u) v \mathrm{~d} x \quad \forall u, v \in \mathcal{H}_{\varepsilon},
$$

and weak solutions to $\left(\mathcal{P}_{\varepsilon}\right)$ are critical points of $I_{\varepsilon}$.

The above properties can be deduced using standard arguments: it is enough to combine the continuous embeddings (2.9) with the Trudinger-Moser bound (1.2), and the following estimates. The growth assumptions $\left(f_{0}\right)$ and $\left(f_{1}\right)$ on nonlinear term $f$ yield, for any $\gamma>0$, $q \geq 0$, and $\delta>0$,

$$
|f(s)| \leq \delta|s|+C(\gamma, q, \delta)|s|^{q}\left(\mathrm{e}^{\gamma s^{2}}-1\right) \quad \forall s \in \mathbb{R} .
$$

Therefore, using the Ambrosetti-Rabinowitz condition $\left(f_{2}\right)$, we get

$$
|F(s)| \leq \delta s^{2}+C(\gamma, q, \delta)|s|^{q+1}\left(\mathrm{e}^{\gamma s^{2}}-1\right) \quad \forall s \in \mathbb{R} .
$$

The functional $I_{\varepsilon}$ has a mountain pass geometry on $\mathcal{H}_{\varepsilon}$. More precisely, 
Lemma 3.1 There exist $\tau=\tau(\varepsilon)$ and $\varrho=\varrho(\varepsilon)>0$ such that $I_{\varepsilon}(u) \geq \tau$ for any $u \in \mathcal{H}_{\varepsilon}$ with $\|u\|_{\varepsilon}=\varrho$, and there exists $e_{\varepsilon} \in H_{\varepsilon}$ with $\left\|e_{\varepsilon}\right\|_{\varepsilon}>\varrho$ such that $I_{\varepsilon}\left(e_{\varepsilon}\right)<0$.

Proof The proof is standard and can be carried out as in [21, Lemma 5.1].

The functional $I_{\varepsilon}$ satisfies the Palais-Smale compactness condition at any level $c \in \mathbb{R}$. We recall that $\left\{u_{n}\right\}_{n} \subset \mathcal{H}_{\varepsilon}$ is a Palais-Smale sequence for $I_{\varepsilon}$ at the level $c \in \mathbb{R}$ (for short, $(P S)_{c}$-sequence) if $I_{\varepsilon}\left(u_{n}\right) \rightarrow c$ and $I_{\varepsilon}^{\prime}\left[u_{n}\right] \rightarrow 0$ as $n \rightarrow+\infty$.

Lemma 3.2 Let $\left\{u_{n}\right\}_{n} \subset \mathcal{H}_{\varepsilon}$ be a $(P S)_{c}$-sequence for $I_{\varepsilon}$ with $c \in \mathbb{R}$.

(I) The sequence $\left\{u_{n}\right\}_{n}$ is bounded in $\mathcal{H}_{\varepsilon}$, and

$$
\sup _{n} \int_{\mathbb{R}^{2}} Q(x) f\left(u_{n}\right) u_{n} \mathrm{~d} x<+\infty .
$$

(II) Up to a subsequence, $u_{n} \rightarrow u_{\varepsilon}$ in $\mathcal{H}_{\varepsilon}$, and $u_{\varepsilon} \in \mathcal{H}_{\varepsilon}$ is a weak solution to $\left(\mathcal{P}_{\varepsilon}\right)$.

Proof The proof of (I) follows by standard arguments as in [21, Lemma 5.3], and we omit it. In order to prove (II), we point out that the boundedness of $\left\{u_{n}\right\}_{n}$ in $\mathcal{H}_{\varepsilon}$ implies the weak convergence $u_{n} \rightarrow u_{\varepsilon}$ in $\mathcal{H}_{\varepsilon}$. Here and below, up to the end of the proof, convergence has to be understood up to a subsequence.

We can rewrite

$$
\left\|u_{n}-u_{\varepsilon}\right\|_{\varepsilon}^{2}=\left(I_{\varepsilon}^{\prime}\left[u_{n}\right]-I_{\varepsilon}^{\prime}\left(u_{\varepsilon}\right)\right)\left(u_{n}-u_{\varepsilon}\right)+\int_{\mathbb{R}^{2}} Q(x)\left(f\left(u_{n}\right)-f\left(u_{\varepsilon}\right)\right)\left(u_{n}-u_{\varepsilon}\right) \mathrm{d} x .
$$

Clearly, $I_{\varepsilon}^{\prime}\left[u_{n}\right]\left(u_{n}-u_{\varepsilon}\right) \rightarrow 0$ as $n \rightarrow+\infty$. Moreover, in view of (3.3), we can apply [16, Lemma 2.1] to conclude that

$$
Q(x) f\left(u_{n}\right) \rightarrow Q(x) f\left(u_{\varepsilon}\right) \quad \text { in } L_{\mathrm{loc}}^{1}\left(\mathbb{R}^{2}\right),
$$

and hence $u_{\varepsilon}$ is a weak solution to $\left(\mathcal{P}_{\varepsilon}\right)$. Therefore, $I_{\varepsilon}^{\prime}\left[u_{\varepsilon}\right]\left(u_{n}-u_{\varepsilon}\right)=0$ for any $n$. The proof is complete if we show that

$$
\lim _{n \rightarrow+\infty} E_{n}=0, \quad \text { where } E_{n}:=\int_{\mathbb{R}^{2}} Q(x)\left(f\left(u_{n}\right)-f\left(u_{\varepsilon}\right)\right)\left(u_{n}-u_{\varepsilon}\right) \mathrm{d} x .
$$

To simplify notations, we will write

$$
\|v\|_{L_{Q}^{2}}^{2}:=\int_{\mathbb{R}^{2}} Q(x) v^{2} \mathrm{~d} x \quad \forall v \in L_{Q}^{2}\left(\mathbb{R}^{2}\right) .
$$

Applying (3.1) and Hölder's inequality, we can estimate

$$
\begin{aligned}
\left|E_{n}\right| \leq & C_{1} \int_{\mathbb{R}^{2}} Q(x)\left(\left|u_{n}\right|+\left|u_{\varepsilon}\right|\right)\left|u_{n}-u_{\varepsilon}\right| \mathrm{d} x \\
& +C_{2} \int_{\mathbb{R}^{2}} Q(x)\left[\left(\mathrm{e}^{\gamma u_{n}^{2}}-1\right)+\left(\mathrm{e}^{\gamma u_{\varepsilon}^{2}}-1\right)\right]\left|u_{n}-u_{\varepsilon}\right| \mathrm{d} x \\
\leq & C_{1}\left(\left\|u_{n}\right\|_{L_{Q}^{2}}+\left\|u_{\varepsilon}\right\|_{L_{Q}^{2}}\right)\left\|u_{n}-u_{\varepsilon}\right\|_{L_{Q}^{2}} \\
& +C_{2}\left[\left(\int_{\mathbb{R}^{2}} Q(x)\left(\mathrm{e}^{2 \gamma u_{n}^{2}}-1\right) \mathrm{d} x\right)^{\frac{1}{2}}+\left(\int_{\mathbb{R}^{2}} Q(x)\left(\mathrm{e}^{2 \gamma u_{\varepsilon}^{2}}-1\right) \mathrm{d} x\right)^{\frac{1}{2}}\right]\left\|u_{n}-u_{\varepsilon}\right\|_{L_{Q}^{2}} .
\end{aligned}
$$

Using the continuity of the embedding $\mathcal{H}_{\varepsilon} \hookrightarrow L_{Q}^{2}\left(\mathbb{R}^{2}\right)$ (see Theorem 2.5), the TrudingerMoser estimate (1.2), and the boundedness of $\left\{u_{n}\right\}_{n}$ in $\mathcal{H}_{\varepsilon}$, we get

$$
\left|E_{n}\right| \leq \tilde{C}_{1}\left\|u_{n}-u_{\varepsilon}\right\|_{L_{Q}^{2}}+C_{2}\left(\int_{\mathbb{R}^{2}} Q(x)\left(\mathrm{e}^{2 \gamma u_{n}^{2}}-1\right) \mathrm{d} x\right)^{\frac{1}{2}}\left\|u_{n}-u_{\varepsilon}\right\|_{L_{Q}^{2}}+\tilde{C}_{2}\left\|u_{n}-u_{\varepsilon}\right\|_{L_{Q}^{2}} .
$$


Since the embedding $\mathcal{H}_{\varepsilon} \hookrightarrow L_{Q}^{2}\left(\mathbb{R}^{2}\right)$ is also compact (see again Theorem 2.5), $u_{n} \rightarrow u_{\varepsilon}$ in $L_{Q}^{2}\left(\mathbb{R}^{2}\right)$. Therefore, the proof of (3.4) is complete if we obtain the existence of $\gamma>0$ such that

$$
\sup _{n} \int_{\mathbb{R}^{2}} Q(x)\left(\mathrm{e}^{2 \gamma u_{n}^{2}}-1\right) \mathrm{d} x<+\infty
$$

and this is a direct consequence of the Trudinger-Moser inequality (1.3). In fact, since there exists $C_{\varepsilon}>0$ such that $\left\|u_{n}\right\|_{\varepsilon}^{2} \leq C_{\varepsilon}$ for any $n$, it is enough to choose $\gamma>0$ sufficiently small so that $2 \gamma C_{\varepsilon} / \varepsilon^{2}<4 \pi$.

Completion of the proof of the existence statement of Theorem $1.2 \mathrm{We}$ introduce the mountain pass level

$c_{\varepsilon}:=\inf _{\gamma \in \Gamma_{\varepsilon}} \sup _{t \in[0,1]} I_{\varepsilon}(\gamma(t)), \quad$ where $\Gamma_{\varepsilon}:=\left\{\gamma \in \mathcal{C}\left([0,1], \mathcal{H}_{\varepsilon}\right) \mid \gamma(0)=0\right.$ and $\left.I_{\varepsilon}(\gamma(1))<0\right\}$.

In view of Lemmas 3.1 and 3.2, the classical mountain pass theorem ensures that $c_{\varepsilon}>0$ is a critical level for $I_{\varepsilon}$. In other words, $I_{\varepsilon}$ has a critical point $u_{\varepsilon} \in \mathcal{H}_{\varepsilon}$ satisfying $I_{\varepsilon}\left(u_{\varepsilon}\right)=c_{\varepsilon}$. Since $c_{\varepsilon}>0, u_{\varepsilon}$ is a nontrivial weak solution for $\left(\mathcal{P}_{\varepsilon}\right)$.

\section{Integral uniform estimates and $L^{2}$-integrability of weak solutions}

In this section, we establish preliminary integral estimates for weak solutions to $\left(\mathcal{P}_{\varepsilon}\right)$ under the assumptions:

- $(V)$ and $(Q)$ hold with $\alpha$ and $\beta$ in the range (1.1),

- $f: \mathbb{R} \rightarrow \mathbb{R}$ is a continuous function with subcritical exponential growth $\left(f_{0}\right)$ satisfying $\left(f_{1}\right)$ and $\left(f_{2}\right)$.

These estimates are uniform with respect to $\varepsilon$, and this aspect is crucial to study the concentration behavior of mountain pass solutions. As a by-product, at the end of this section, we will show that any weak solution $u_{\varepsilon} \in \mathcal{H}_{\varepsilon}$ for $\left(\mathcal{P}_{\varepsilon}\right)$ satisfies $u_{\varepsilon} \in L^{2}\left(\mathbb{R}^{2}\right)$, and hence $u_{\varepsilon} \in H^{1}\left(\mathbb{R}^{2}\right)$. We will follow the arguments introduced in [6, Lemma 17 and Lemma 18], see also [26, Section 3] and [21, Section 6].

Lemma 4.1 Let $M>0$, and $\varepsilon_{0} \in(0,1]$. For any $\varepsilon \in\left(0, \varepsilon_{0}\right)$, let $u_{\varepsilon} \in \mathcal{H}_{\varepsilon}$ be a weak solution to $\left(\mathcal{P}_{\varepsilon}\right)$ satisfying

$$
\left\|u_{\varepsilon}\right\|_{\varepsilon}^{2} \leq M \varepsilon^{2} .
$$

Then there exists $R_{0}>0$ such that, for any $n \in \mathbb{N}$ with $R_{n, \varepsilon}:=\varepsilon n^{2 /(2-\alpha)} \geq R_{0}$, we have

$$
\int_{B_{R_{n+1, \varepsilon}^{c}}^{c}}\left(\varepsilon^{2}\left|\nabla u_{\varepsilon}\right|^{2}+V(x) u_{\varepsilon}^{2}\right) \mathrm{d} x \leq \frac{3}{4} \int_{B_{R_{n, \varepsilon}}^{c}}\left(\varepsilon^{2}\left|\nabla u_{\varepsilon}\right|^{2}+V(x) u_{\varepsilon}^{2}\right) \mathrm{d} x .
$$

Proof Let $\chi_{n, \varepsilon}: \mathbb{R}^{2} \rightarrow[0,1]$ be a piecewise affine function such that

$$
\chi_{n, \varepsilon} \equiv 0 \text { in } B_{R_{n, \varepsilon}} \text { and } \chi_{n, \varepsilon} \equiv 1 \text { in } B_{R_{n+1, \varepsilon}}^{c} .
$$

Arguing as in [6, Lemma 17], we get the estimate

$$
\varepsilon^{2}\left|\nabla \chi_{n, \varepsilon}(x)\right|^{2} \leq V(x) \quad \forall x \in \mathbb{R}^{2} .
$$


By construction $\chi_{n, \varepsilon} u_{\varepsilon} \in H_{V}^{1}\left(\mathbb{R}^{2}\right)$,

$$
\int_{B_{R_{n+1, \varepsilon}}^{\mathrm{c}}}\left(\varepsilon^{2}\left|\nabla u_{\varepsilon}\right|^{2}+V(x) u_{\varepsilon}^{2}\right) \mathrm{d} x \leq \int_{B_{R_{n, \varepsilon}}^{\mathrm{c}}} \chi_{n, \varepsilon}\left(\varepsilon^{2}\left|\nabla u_{\varepsilon}\right|^{2}+V(x) u_{\varepsilon}^{2}\right) \mathrm{d} x,
$$

and we can compute

$$
\left\langle u_{\varepsilon}, \chi_{n, \varepsilon} u_{\varepsilon}\right\rangle_{\varepsilon}=\int_{B_{R_{n, \varepsilon}}^{\mathrm{c}}} \chi_{n, \varepsilon}\left(\varepsilon^{2}\left|\nabla u_{\varepsilon}\right|^{2}+V(x) u_{\varepsilon}^{2}\right) d x+\varepsilon^{2} \int_{B_{R_{n, \varepsilon}}^{\mathrm{c}}} u_{\varepsilon} \nabla u_{\varepsilon} \cdot \nabla \chi_{n, \varepsilon} \mathrm{d} x .
$$

Moreover, taking $\chi_{n, \varepsilon} u_{\varepsilon} \in \mathcal{H}_{\varepsilon}$ as test function, we obtain

$$
\left\langle u_{\varepsilon}, \chi_{n, \varepsilon} u_{\varepsilon}\right\rangle_{\varepsilon}-\int_{\mathbb{R}^{2}} Q(x) f\left(u_{\varepsilon}\right) \chi_{n, \varepsilon} u_{\varepsilon} \mathrm{d} x=0 .
$$

Therefore, we can estimate

$$
\begin{gathered}
\int_{B_{R_{n, \varepsilon}}^{\mathrm{c}}} \chi_{n, \varepsilon}\left(\varepsilon^{2}\left|\nabla u_{\varepsilon}\right|^{2}+V(x) u_{\varepsilon}^{2}\right) \mathrm{d} x=\int_{B_{R_{n, \varepsilon}}^{\mathrm{c}}} Q(x) f\left(u_{\varepsilon}\right) \chi_{n} u_{\varepsilon} \mathrm{d} x-\varepsilon^{2} \int_{B_{R_{n, \varepsilon}}^{\mathrm{c}}} u_{\varepsilon} \nabla u_{\varepsilon} \cdot \nabla \chi_{n, \varepsilon} \mathrm{d} x \\
\quad \leq \int_{B_{R_{n, \varepsilon}}^{\mathrm{c}}} Q(x) f\left(u_{\varepsilon}\right) u_{\varepsilon} \mathrm{d} x+\frac{\varepsilon^{2}}{2}\left(\int_{B_{R_{n, \varepsilon}}^{\mathrm{c}}}\left|\nabla u_{\varepsilon}\right|^{2} \mathrm{~d} x+\int_{B_{R_{n, \varepsilon}}^{\mathrm{c}}}\left|\nabla \chi_{n, \varepsilon}\right|^{2} u_{\varepsilon}^{2} \mathrm{~d} x\right) \\
\leq \int_{B_{R_{n, \varepsilon}}^{\mathrm{c}}} Q(x) f\left(u_{\varepsilon}\right) u_{\varepsilon} \mathrm{d} x+\frac{1}{2} \int_{B_{R_{n, \varepsilon}}^{\mathrm{c}}}\left(\varepsilon^{2}\left|\nabla u_{\varepsilon}\right|^{2}+V(x) u_{\varepsilon}^{2}\right) \mathrm{d} x .
\end{gathered}
$$

The proof is complete if we establish the existence of $R_{0}>0$ such that, for any $n \in \mathbb{N}$ with $R_{n, \varepsilon} \geq R_{0}$, the following inequality holds

$$
\int_{B_{R_{n, \varepsilon}}^{\mathrm{c}}} Q(x) f\left(u_{\varepsilon}\right) u_{\varepsilon} \mathrm{d} x \leq \frac{1}{4} \int_{B_{R_{n, \varepsilon}}^{\mathrm{c}}}\left(\varepsilon^{2}\left|\nabla u_{\varepsilon}\right|^{2}+V(x) u_{\varepsilon}^{2}\right) \mathrm{d} x .
$$

Applying (3.1) and Hölder's inequality, for any fixed $\gamma>0$ we have

$$
\begin{aligned}
\int_{B_{R_{n, \varepsilon}}^{\mathrm{c}}} Q(x) f\left(u_{\varepsilon}\right) u_{\varepsilon} \mathrm{d} x \leq & \int_{B_{R_{n, \varepsilon}}^{\mathrm{c}}} Q(x) u_{\varepsilon}^{2} \mathrm{~d} x \\
& +C(\gamma)\left(\int_{B_{R_{n, \varepsilon}}^{\mathrm{c}}} Q(x) u_{\varepsilon}^{2} \mathrm{~d} x\right)^{\frac{1}{2}}\left(\int_{B_{R_{n, \varepsilon}}^{\mathrm{c}}} Q(x)\left(\mathrm{e}^{2 \gamma u_{\varepsilon}^{2}}-1\right) \mathrm{d} x\right)^{\frac{1}{2}} .
\end{aligned}
$$

To estimate the exponential integral, we choose $\gamma \in(0, \bar{\gamma} / 2)$ and $R_{0} \geq \bar{R}$, where $\bar{\gamma}$ and $\bar{R}$ are given by Lemma 2.6 with $\eta=1$. With this choice of $\gamma$ and $R_{0}$, for any $n \in \mathbb{N}$ satisfying $R_{n, \varepsilon} \geq R_{0}$, we have

$$
\int_{B_{R_{n, \varepsilon}}^{\mathrm{c}}} Q(x)\left(\mathrm{e}^{2 \gamma u_{\varepsilon}^{2}}-1\right) \mathrm{d} x \leq \int_{B_{R_{n, \varepsilon}}^{\mathrm{c}}}\left(\varepsilon^{2}\left|\nabla u_{\varepsilon}\right|^{2}+V(x) u_{\varepsilon}^{2}\right) \mathrm{d} x .
$$

To estimate the weighted $L^{2}$-norm, we point out that, for any $n \in \mathbb{N}$ with $R_{n, \varepsilon} \geq R_{0}$, we have

$$
\sup _{x \in B_{R_{n, \varepsilon}}^{\mathrm{c}}} \frac{Q(x)}{V(x)} \leq \sup _{x \in B_{R_{0}}^{\mathrm{c}}} \frac{Q(x)}{V(x)} \leq \sup _{x \in B_{R_{0}}^{\mathrm{c}}} \frac{b}{a} \frac{1+|x|^{\alpha}}{1+|x|^{\beta}} \leq \frac{b}{a} \frac{1+\left|R_{0}\right|^{\alpha}}{1+\left|R_{0}\right|^{\beta}}=: \mathcal{B}\left(R_{0}\right),
$$

where we used assumptions $(V)$ and $(Q)$ with $\alpha$ and $\beta$ in the range (1.1). Hence,

$$
\int_{B_{R_{n, \varepsilon}}^{\mathrm{c}}} Q(x) u_{\varepsilon}^{2} \mathrm{~d} x \leq \mathcal{B}\left(R_{0}\right) \int_{B_{R_{n, \varepsilon}}^{\mathrm{c}}} V(x) u_{\varepsilon}^{2} \mathrm{~d} x,
$$


and it is important to point out that

$$
\lim _{R_{0} \rightarrow+\infty} \mathcal{B}\left(R_{0}\right)=\lim _{R_{0} \rightarrow+\infty} \frac{b}{a} R_{0}^{\alpha-\beta}=0 .
$$

Combining (4.3) and (4.4), we obtain

$\int_{B_{R_{n, \varepsilon}}^{\mathrm{c}}} Q(x) f\left(u_{\varepsilon}\right) u_{\varepsilon} \mathrm{d} x \leq\left[\left[\mathcal{B}\left(R_{0}\right)\right]^{\frac{1}{2}}+C(\gamma)\right]\left[\mathcal{B}\left(R_{0}\right)\right]^{\frac{1}{2}} \int_{B_{R_{n, \varepsilon}}^{\mathrm{c}}}\left(\varepsilon^{2}\left|\nabla u_{\varepsilon}\right|^{2}+V(x) u_{\varepsilon}^{2}\right) \mathrm{d} x$.

Since $\gamma \in(0, \bar{\gamma} / 2)$ is fixed, we can choose $R_{0} \geq \bar{R}$ sufficiently large so that

$$
\left[\left[\mathcal{B}\left(R_{0}\right)\right]^{\frac{1}{2}}+C(\gamma)\right]\left[\mathcal{B}\left(R_{0}\right)\right]^{\frac{1}{2}} \leq \frac{1}{4}
$$

and this completes the proof of (4.2).

Lemma 4.2 Let $M>0$, and $\varepsilon_{0} \in(0,1]$. For any $\varepsilon \in\left(0, \varepsilon_{0}\right)$, let $u_{\varepsilon} \in \mathcal{H}_{\varepsilon}$ be a weak solution to $\left(\mathcal{P}_{\varepsilon}\right)$ satisfying (4.1). Then there exists $R_{0}>0$ and a constant $C=C(M)>0$ such that

$\int_{B_{\varrho}^{c}}\left(\varepsilon^{2}\left|\nabla u_{\varepsilon}\right|^{2}+V(x) u_{\varepsilon}^{2}\right) \mathrm{d} x \leq C \varepsilon^{2} \exp \left\{-|\log (3 / 4)| \varepsilon^{-(2-\alpha) / 2}\left[\varrho^{(2-\alpha) / 2}-R^{(2-\alpha) / 2}\right]\right\}$, for any $R \geq R_{0}$ and $\varrho>2 R$.

Proof Let $R_{0}$ and $\left\{R_{n, \varepsilon}\right\}_{n}$ be as in Lemma 4.1. If $R \geq R_{0}$ and $\varrho>2 R$, then we have the existence of two positive integers $\bar{n}>\tilde{n}$ such that

$$
R_{\tilde{n}, \varepsilon} \leq R \leq R_{\tilde{n}+1, \varepsilon} \quad \text { and } \quad R_{\bar{n}-1, \varepsilon} \leq \varrho \leq R_{\bar{n}, \varepsilon} .
$$

By definition of $R_{n, \varepsilon}$, it is easy to see that

$$
\bar{n}-\tilde{n} \geq \varepsilon^{-(2-\alpha) / 2}\left(\varrho^{(2-\alpha) / 2}-R^{(2-\alpha) / 2}\right)>(R / \varepsilon)^{(2-\alpha) / 2}\left(2^{(2-\alpha) / 2}-1\right)>2
$$

provided $R_{0}$ is sufficiently large, and it can still be chosen independently of $\varepsilon \in(0,1]$. Therefore, $R_{\bar{n}-1, \varepsilon} \geq R_{\tilde{n}+1, \varepsilon} \geq R \geq R_{0}$, and in view of (4.1), we can apply Lemma 4.1. In this way, we can estimate

$$
\begin{aligned}
& \int_{B_{\varrho}^{\mathrm{c}}}(\left.\varepsilon^{2}\left|\nabla u_{\varepsilon}\right|^{2}+V(x) u_{\varepsilon}^{2}\right) \mathrm{d} x \leq \int_{B_{R_{\bar{n}-1, \varepsilon}}^{\mathrm{c}}}\left(\varepsilon^{2}\left|\nabla u_{\varepsilon}\right|^{2}+V(x) u_{\varepsilon}^{2}\right) \mathrm{d} x \\
& \quad \leq(3 / 4)^{\bar{n}-\tilde{n}-2} \int_{B_{R}^{\mathrm{c}}}\left(\varepsilon^{2}\left|\nabla u_{\varepsilon}\right|^{2}+V(x) u_{\varepsilon}^{2}\right) \mathrm{d} x \\
& \quad \leq(4 / 3)^{2} \exp \left\{-|\log (3 / 4)| \varepsilon^{-(2-\alpha) / 2}\left(\varrho^{(2-\alpha) / 2}-R^{(2-\alpha) / 2}\right)\right\} \int_{B_{R}^{\mathrm{c}}}\left(\varepsilon^{2}\left|\nabla u_{\varepsilon}\right|^{2}+V(x) u_{\varepsilon}^{2}\right) \mathrm{d} x,
\end{aligned}
$$

and the desired result follows again from (4.1).

We will show that the mountain pass solutions found in Theorem 1.2 satisfy (4.1) for any $\varepsilon>0$ sufficiently small, see Corollary 5.4. Therefore, the $L^{2}$-uniform bound (1.4) for mountain pass solutions to $\left(\mathcal{P}_{\varepsilon}\right)$ is a consequence of the following result.

Proposition 4.3 Let $M>0$, and $\varepsilon_{0} \in(0,1]$. For any $\varepsilon \in\left(0, \varepsilon_{0}\right)$, let $u_{\varepsilon} \in \mathcal{H}_{\varepsilon}$ be a weak solution to $\left(\mathcal{P}_{\varepsilon}\right)$ satisfying (4.1). Then there exists $C>0$ such that

$$
\int_{\mathbb{R}^{2}} u_{\varepsilon}^{2} \mathrm{~d} x \leq C \varepsilon^{2} \quad \forall \varepsilon \in\left(0, \varepsilon_{0}\right) .
$$


Proof First, we recall that $L_{V}^{2}\left(\mathbb{R}^{2}\right) \hookrightarrow L_{\text {loc }}^{2}\left(\mathbb{R}^{2}\right)$, and for any $\Omega \subset \mathbb{R}^{2}$ bounded there exists $C(\Omega)>0$ such that

$$
\int_{\Omega} u^{2} \mathrm{~d} x \leq C(\Omega)\|u\|_{\varepsilon}^{2} \quad \forall u \in H_{V}^{1}\left(\mathbb{R}^{2}\right), \forall \varepsilon>0 .
$$

Combining (4.1) with (4.6), for any $\Omega \subset \mathbb{R}^{2}$ bounded we have

$$
\int_{\Omega} u_{\varepsilon}^{2} \mathrm{~d} x \leq C(\Omega) M \varepsilon^{2} \quad \forall \varepsilon \in\left(0, \varepsilon_{0}\right) .
$$

If we obtain a suitable uniform estimate of the $L^{2}$-norm of $u_{\varepsilon}$ on $B_{2}^{\mathrm{c}}$, then the proof is complete, and to this aim, the idea is to apply Lemma 4.2. If $r \geq 2$ and $y \in B_{2 r}^{c}$, we have

$$
\sup _{x \in B(y, r)} \frac{1+|x|^{\alpha}}{a|y|^{\alpha}} \leq \frac{1+(r+|y|)^{\alpha}}{a|y|^{\alpha}} \leq \frac{1+\left(\frac{3}{2}|y|\right)^{\alpha}}{a|y|^{\alpha}} \leq \sup _{y \in B_{4}^{c}} \frac{1+\left(\frac{3}{2}|y|\right)^{\alpha}}{a|y|^{\alpha}}=: C(\alpha)<+\infty
$$

and hence

$$
\int_{B(y, r)} u_{\varepsilon}^{2} \mathrm{~d} x \leq \int_{B(y, r)} \frac{1+|x|^{\alpha}}{a} V(x) u_{\varepsilon}^{2} \mathrm{~d} x \leq C(\alpha)|y|^{\alpha} \int_{B_{|y| / 2}^{\mathrm{c}}} V(x) u_{\varepsilon}^{2} \mathrm{~d} x,
$$

where we also used $(V)$ and the inclusion $B(y, r) \subseteq B_{|y| / 2}^{\mathrm{c}}$.

If $R_{0}>1$ is given by Lemma 4.2, and we choose $r>2 R_{0}$, then applying Lemma 4.2 we get

$$
\int_{B(y, r)} u_{\varepsilon}^{2} \mathrm{~d} x \leq \tilde{C}(\alpha)|y|^{\alpha} \varepsilon^{2} \exp \left\{-\bar{C}(\alpha)|\log (3 / 4)| \varepsilon^{-(2-\alpha) / 2}|y|^{(2-\alpha) / 2}\right\} .
$$

Let $m \in \mathbb{N}$ and $y_{i} \in B_{2}^{c}$ with $i \in\{1, \ldots, m\}$ be such that

$$
B_{5} \backslash B_{2} \subset \bigcup_{i=1}^{m} B\left(y_{i}, 1\right)
$$

and let $y_{i, k}:=2^{k} y_{i}$. If $K_{0}$ is a positive integer such that $2^{K_{0}}>2 R_{0}$ then, using (4.8) with $r=2^{k}$ and $y=y_{i, k}$, we obtain

$$
\int_{B\left(y_{i, k}, 2^{k}\right)} u_{\varepsilon}^{2} \mathrm{~d} x \leq \tilde{C}(\alpha) \varepsilon^{2}\left|y_{i, k}\right|^{\alpha} \exp \left\{-\bar{C}(\alpha)|\log (3 / 4)| \varepsilon^{-(2-\alpha) / 2}\left|y_{i, k}\right|^{(2-\alpha) / 2}\right\} \quad \forall k \geq K_{0},
$$

and

$$
\begin{aligned}
\int_{B_{2}^{\mathrm{c}}} u_{\varepsilon}^{2} \mathrm{~d} x & \leq \sum_{k=0}^{+\infty} \int_{2^{k} B_{5} \backslash B_{2}} u_{\varepsilon}^{2} \mathrm{~d} x \leq \sum_{i=1}^{m} \sum_{k=0}^{+\infty} \int_{B\left(y_{i, k}, 2^{k}\right)} u_{\varepsilon}^{2} \mathrm{~d} x \leq \sum_{i=1}^{m} \sum_{k=0}^{K_{0}-1} \int_{B\left(y_{i, k}, 2^{k}\right)} u_{\varepsilon}^{2} \mathrm{~d} x \\
+ & \tilde{C}(\alpha) \varepsilon^{2} \sum_{i=1}^{m} \sum_{k=K_{0}}^{+\infty}\left\{\left|y_{i, k}\right|^{\alpha} \exp \left[-\bar{C}(\alpha)|\log (3 / 4)| \varepsilon^{-(2-\alpha) / 2}\left|y_{i, k}\right|^{(2-\alpha) / 2}\right]\right\} .
\end{aligned}
$$

Since $\alpha \in(0,2)$, the series on the right-hand side is finite and uniformly bounded for any $\varepsilon \in\left(0, \varepsilon_{0}\right)$. This combined with (4.7) enables us to conclude that (4.5) holds.

We conclude this section showing that any weak solution $u_{\varepsilon} \in \mathcal{H}_{\varepsilon}$ for $\left(\mathcal{P}_{\varepsilon}\right)$ is a bound state solution, i.e., it has finite $L^{2}$-norm. 
Proposition 4.4 Any weak solution $u_{\varepsilon} \in \mathcal{H}_{\varepsilon}$ to $\left(\mathcal{P}_{\varepsilon}\right)$ satisfies $u_{\varepsilon} \in L^{2}\left(\mathbb{R}^{2}\right)$, and hence $u_{\varepsilon} \in H^{1}\left(\mathbb{R}^{2}\right)$.

Proof Let $\varepsilon>0$ be fixed, and let $u_{\varepsilon}$ be any weak solution to $\left(\mathcal{P}_{\varepsilon}\right)$. Clearly, $u_{\varepsilon}$ satisfies (4.1) with $M=M(\varepsilon):=\left\|u_{\varepsilon}\right\|_{\varepsilon}^{2} / \varepsilon^{2}$, and Lemma 4.1 and Lemma 4.2 still hold for some $R_{0}>0$ depending on $\varepsilon$. Therefore, we can argue as in the proof of Proposition 4.3 to obtain (4.8), and hence (4.9). Since $\alpha \in(0,2)$, the series on the right-hand side of (4.9) is finite, and this combined with (4.6) enables us to conclude that $u_{\varepsilon} \in L^{2}\left(\mathbb{R}^{2}\right)$.

In view of Proposition 4.3 (see also Corollary 5.4) and Proposition 4.4, the proof of Theorem 1.2 is complete.

\section{Proof of Theorem 1.3}

In this section, we consider the mountain pass solutions $u_{\varepsilon}$ found in Theorem 1.2, and we assume:

- $(V)$ and $(Q)$ hold with $\alpha$ and $\beta$ in the range (1.1),

- $f \in \mathcal{C}^{0, \sigma}(\mathbb{R})$ with $\sigma \in(0,1)$ satisfies $\left(f_{0}\right),\left(f_{1}\right)$, and $\left(f_{2}\right)$.

Since $u_{\varepsilon}$ is a weak solution to $\left(\mathcal{P}_{\varepsilon}\right), u_{\varepsilon}$ is also a classical solution and $u_{\varepsilon} \in \mathcal{C}^{2}\left(\mathbb{R}^{2}\right)$. This can be shown through interior $L^{2}$-estimates, the classical Sobolev embeddings, and Schauder estimates (see, e.g., [34, Proposition 2.15]).

Since $u_{\varepsilon} \in H^{1}\left(\mathbb{R}^{2}\right)$, by standard arguments (see, e.g., [34, Remark 2.34]),

$$
\lim _{|x| \rightarrow+\infty} u_{\varepsilon}(x)=0
$$

and hence, by the strong maximum principle, $u_{\varepsilon}>0$ in $\mathbb{R}^{2}$.

The proof of Theorem 1.3 will be carried out by means of several steps.

\subsection{The limiting problem}

First, we recall some known results about the limiting problem associated with $\left(\mathcal{P}_{\varepsilon}\right)$. If $\xi \in \mathbb{R}^{2}$ is appropriately chosen, then the limiting equation for $\left(\mathcal{P}_{\varepsilon}\right)$ is of the form

$$
-\Delta U+V(\xi) U=Q(\xi) f(U) \text { in } \mathbb{R}^{2} .
$$

Let $L_{\xi}: H^{1}\left(\mathbb{R}^{2}\right) \rightarrow \mathbb{R}$ be the related energy functional, i.e.,

$$
L \xi(U)=\frac{1}{2} \int_{\mathbb{R}^{2}}|\nabla U|^{2} \mathrm{~d} x+\frac{V(\xi)}{2} \int_{\mathbb{R}^{2}} U^{2} \mathrm{~d} x-Q(\xi) \int_{\mathbb{R}^{2}} F(U) \mathrm{d} x .
$$

Clearly, $L_{\xi}$ is of class $C^{1}$ and its critical points are solutions to (5.1). The existence of a least energy solution for equation (5.1) was established in [7], and we denote by $m_{\xi}$ the corresponding least energy level, i.e.,

$$
m_{\xi}:=\inf \left\{L_{\xi}(u): u \in H^{1}\left(\mathbb{R}^{2}\right) \backslash\{0\} \text { is a solution to (5.1) }\right\}
$$

Next, we recall the following characterization of the least energy level. 
Theorem 5.1 [24] Let $\xi \in \mathbb{R}^{2}$. The least energy level $m_{\xi}$ of the functional $L_{\xi}$ coincides with the mountain pass level, i.e.,

$$
m_{\xi}=\inf _{\gamma \in \Gamma_{\xi}} \max _{t \in[0,1]} L_{\xi}(\gamma(t)),
$$

where $\Gamma_{\xi}=\left\{\gamma \in C\left([0,1], H^{1}\left(\mathbb{R}^{2}\right)\right): \gamma(0)=0\right.$ and $\left.L_{\xi}(\gamma(1))<0\right\}$. Moreover, for any least energy solution $U \in H^{1}\left(\mathbb{R}^{2}\right)$ of (5.1), there exists a path $\gamma \in \Gamma_{\xi}$ such that

$$
U \in \gamma([0,1]) \text { and } \max _{t \in[0,1]} L_{\xi}(\gamma(t))=L_{\xi}(U)=m_{\xi} .
$$

Remark 5.2 Exploiting the properties of dilations, if we set

$$
U_{\lambda}(x):=U(\lambda x), \quad x \in \mathbb{R}^{2}, \lambda>0,
$$

then $L_{\xi}(U)=\tilde{L}_{\xi}\left(U_{\lambda}\right)$ where $\tilde{L}_{\xi}$ is the auxiliary functional

$$
\tilde{L}_{\xi}(U):=\frac{1}{2} \int_{\mathbb{R}^{2}}|\nabla U|^{2} \mathrm{~d} x+\frac{\mathcal{A}(\xi)}{2} \int_{\mathbb{R}^{2}} U^{2} \mathrm{~d} x-\int_{\mathbb{R}^{2}} F(U) \mathrm{d} x,
$$

defined by means of $\mathcal{A}:=V / Q$. It is worth pointing out that the mountain pass level $m_{\xi}$ of $L_{\xi}$ coincides with the mountain pass level of the auxiliary functional $\tilde{L}_{\xi}$. Hence, using the definition of $\tilde{L}_{\xi}$, we deduce

$$
\text { if } \mathcal{A}\left(\xi_{1}\right)<\mathcal{A}\left(\xi_{2}\right) \text {, for some } \xi_{1}, \xi_{2} \in \mathbb{R}^{2} \text {, then } m_{\xi_{1}}<m_{\xi_{2}} \text {. }
$$

We also observe that, in view of $(V)$ and $(Q)$, since $\beta>\alpha$, the function $\mathcal{A}$ has a minimum point $\xi_{0} \in \mathbb{R}^{2}$. So $m_{\xi_{0}} \leq m_{\xi}$ for all $\xi \in \mathbb{R}^{2}$.

\subsection{Uniform estimate of the mountain pass level}

Let $c_{\varepsilon}$ be the mountain pass critical level associated with $\left(\mathcal{P}_{\varepsilon}\right)$ defined in (3.5).

Proposition 5.3 For any $\xi \in \mathbb{R}^{2}$, there exists $\varepsilon_{0}=\varepsilon_{0}(\xi)>0$ such that

$$
\varepsilon^{-2} c_{\varepsilon}=\varepsilon^{-2} I_{\varepsilon}\left(u_{\varepsilon}\right) \leq m_{\xi}+o_{\xi}(1), \quad \forall \varepsilon \in\left(0, \varepsilon_{0}\right)
$$

where $\lim _{\varepsilon \rightarrow 0} o_{\xi}(1)=0$. In particular, there exists $C>0$ such that $c_{\varepsilon} \leq C \varepsilon^{2}$ for all $\varepsilon \in\left(0, \varepsilon_{0}\right)$.

Proof Let $\xi \in \mathbb{R}^{2}$. We consider a least energy solution $U \in H^{1}\left(\mathbb{R}^{2}\right)$ for (5.1) and a path $\gamma \in \Gamma_{\xi}$ satisfying (5.3). Let $\varphi \in C_{0}^{\infty}(B(\xi, 2))$ be a cutoff function such that

$$
0 \leq \varphi \leq 1 \text { in } B(\xi, 2) \text { and } \varphi \equiv 1 \text { in } B(\xi, 1),
$$

For any $\varepsilon>0$ and $t \in[0,1]$, we define

$$
\varphi_{\varepsilon, t}(x)=\varphi(x) \gamma(t)\left(\frac{x-\xi}{\varepsilon}\right), \quad x \in \mathbb{R}^{2} .
$$

Since $\gamma(t) \in H^{1}\left(\mathbb{R}^{2}\right)$, we get $\varphi_{\varepsilon, t} \in H^{1}\left(\mathbb{R}^{2}\right) \subset \mathcal{H}_{\varepsilon}$, and we can compute

$$
\begin{aligned}
\int_{\mathbb{R}^{2}}\left|\nabla \varphi_{\varepsilon, t}\right|^{2} \mathrm{~d} x= & \int_{\mathbb{R}^{2}}|\nabla \varphi(\varepsilon y+\xi)|^{2}[\gamma(t)(y)]^{2} d y+2 \int_{\mathbb{R}^{2}} \nabla \varphi(\varepsilon y+\xi) \cdot(\nabla \gamma(t)(y)) d y \\
& +\int_{\mathbb{R}^{2}} \varphi^{2}(\varepsilon y+\xi)|\nabla \gamma(t)(y)|^{2} d y \\
= & \int_{\mathbb{R}^{2}}|\nabla \gamma(t)(y)|^{2} d y+o_{\xi}(1),
\end{aligned}
$$


$\frac{1}{\varepsilon^{2}} \int_{\mathbb{R}^{2}} V(x) \varphi_{\varepsilon, t}^{2} \mathrm{~d} x=\int_{\mathbb{R}^{2}} V(\varepsilon y+\xi) \varphi(\varepsilon y+\xi)^{2}[\gamma(t)(y)]^{2} d y=V(\xi) \int_{\mathbb{R}^{2}}[\gamma(t)(y)]^{2} d y+o \xi(1)$,

and

$$
\begin{aligned}
\frac{1}{\varepsilon^{2}} \int_{\mathbb{R}^{2}} Q(x) F\left(\varphi_{\varepsilon, t}\right) \mathrm{d} x & =\int_{\mathbb{R}^{2}} Q(\varepsilon y+\xi) F(\varphi(\varepsilon y+\xi) \gamma(t)(y)) d y \\
& =Q(\xi) \int_{\mathbb{R}^{2}} F(\gamma(t)(y)) d y+o_{\xi}(1),
\end{aligned}
$$

where $\lim _{\varepsilon \rightarrow 0} o_{\xi}(1)=0$ uniformly in $t \in[0,1]$. Then

$$
\begin{aligned}
\varepsilon^{-2} I_{\varepsilon}\left(\varphi_{\varepsilon, t}\right) & =\frac{1}{2} \int_{\mathbb{R}^{2}}\left|\nabla \varphi_{\varepsilon, t}\right|^{2} \mathrm{~d} x+\frac{1}{2 \varepsilon^{2}} \int_{\mathbb{R}^{2}} V(x) \varphi_{\varepsilon, t}^{2} \mathrm{~d} x-\frac{1}{\varepsilon^{2}} \int_{\mathbb{R}^{2}} Q(x) F\left(\varphi_{\varepsilon, t}\right) \mathrm{d} x \\
& =L_{\xi}(\gamma(t))+o_{\xi}(1) \leq m_{\xi}+o_{\xi}(1) .
\end{aligned}
$$

By assumption $L_{\xi}(\gamma(1))<0$, hence $I_{\varepsilon}\left(\varphi_{\varepsilon, 1}\right)<0$, for sufficiently small values of $\varepsilon>0$. Therefore, $\varphi_{\varepsilon, t} \in \Gamma_{\varepsilon}$, for any $0<\varepsilon<<1$, and we get the estimate

$$
\varepsilon^{-2} c_{\varepsilon} \leq \varepsilon^{-2} \max _{t \in[0,1]} I_{\varepsilon}\left(\varphi_{\varepsilon, t}\right) \leq m_{\xi}+o_{\xi}(1),
$$

as desired.

As a consequence of the above proposition, we have

Corollary 5.4 There exist $\varepsilon_{0}>0$ and $M>0$ satisfying

$$
\left\|u_{\varepsilon}\right\|_{\varepsilon}^{2} \leq M \varepsilon^{2} \quad \forall \varepsilon \in\left(0, \varepsilon_{0}\right) .
$$

Proof In fact, since $u_{\varepsilon}$ is a mountain pass solution for $\left(\mathcal{P}_{\varepsilon}\right)$ and $f$ satisfies $\left(f_{2}\right)$, we have

$$
c_{\varepsilon}=I_{\varepsilon}\left(u_{\varepsilon}\right)-\frac{1}{\mu} I_{\varepsilon}^{\prime}\left[u_{\varepsilon}\right]\left(u_{\varepsilon}\right) \geq\left(\frac{\mu-2}{2 \mu}\right)\left\|u_{\varepsilon}\right\|_{\varepsilon}^{2}
$$

and the result follows from Proposition 5.3.

\subsection{Pointwise uniform exponential decay of mountain pass solutions}

Our next result shows that, at least for sufficiently small values of $\varepsilon>0$, the mountain pass solutions $u_{\varepsilon}$ have a pointwise uniform exponential decay at infinity determined by $\alpha \in(0,2)$.

Theorem 5.5 [Uniform decay estimate] There exists $\varepsilon_{0}>0$ such that $u_{\varepsilon} \in L^{\infty}\left(\mathbb{R}^{2}\right)$ for any $\varepsilon \in\left(0, \varepsilon_{0}\right)$. Moreover, there exist $C>0, d>1$ and $R>0$ such that for any $\varepsilon \in\left(0, \varepsilon_{0}\right)$ and any $|x| \geq 2 R+2$ it holds

$$
\left|u_{\varepsilon}(x)\right| \leq C \varepsilon^{1-d}|x|^{\alpha / 2} \exp \left\{-\frac{1}{2}\left|\log \frac{3}{4}\right|(2 \varepsilon)^{-(2-\alpha) / 2}\left[|x|^{(2-\alpha) / 2}-(2 R)^{(2-\alpha) / 2}\right]\right\} .
$$

Proof We already know that $u_{\varepsilon} \in L_{\mathrm{loc}}^{\infty}\left(\mathbb{R}^{2}\right)$, and clearly this property combined with (5.5) yields $u \in L^{\infty}\left(\mathbb{R}^{2}\right)$. Therefore, the rest of the proof will be devoted to the pointwise uniform bound (5.5). To simplify notations, let

$$
g_{\varepsilon}(x)=Q(x) f\left(u_{\varepsilon}(x)\right), \quad x \in \mathbb{R}^{2} .
$$


From standard interior $L^{2}$-estimates (see, e.g., [23, Theorem 9.11]), for any $\varepsilon>0$ and any $y \in \mathbb{R}^{2}$, we have the local inequality

$$
\left\|u_{\varepsilon}\right\|_{H^{2}(B(y, 1))} \leq C \varepsilon^{-d}\left[\left\|u_{\varepsilon}\right\|_{L^{2}(B(y, 2))}+\left\|g_{\varepsilon}\right\|_{L^{2}(B(y, 2))}\right],
$$

where $d>1$ is a universal constant, and $C=C(A)>0$ with $A$ given by $(V)-$ in other words, the constant $C$ depends only on the norm of $V$ in $L^{\infty}\left(\mathbb{R}^{2}\right)$. Recalling that $H_{\text {loc }}^{2}\left(\mathbb{R}^{2}\right) \hookrightarrow L_{\text {loc }}^{\infty}\left(\mathbb{R}^{2}\right)$, we get

$$
\left\|u_{\varepsilon}\right\|_{L^{\infty}(B(y, 1))} \leq C^{\prime}\left\|u_{\varepsilon}\right\|_{H^{2}(B(y, 1))} \leq C^{\prime \prime} \varepsilon^{-d}\left[\left\|u_{\varepsilon}\right\|_{L^{2}(B(y, 2))}+\left\|g_{\varepsilon}\right\|_{L^{2}(B(y, 2))}\right] .
$$

The idea is to estimate the right-hand side of (5.6) in terms of the $\|\cdot\|_{\varepsilon}$-norm of $u_{\varepsilon}$ in the exterior of a large ball. With this in mind, we consider $|y|>2 R+2$, with $R>1$, so that $B(y, 2) \subset B_{|y|-2}^{c} \subset B_{2 R}^{c}$.

First, recalling that $(V)$ implies $a \leq 5 V(x)|y|^{\alpha}$ for any $x \in B(y, 2)$ and $|y|>2$, we have

$$
\int_{B(y, 2)} u_{\varepsilon}^{2}(x) \mathrm{d} x \leq \frac{5}{a}|y|^{\alpha} \int_{B(y, 2)} V(x) u_{\varepsilon}^{2}(x) \mathrm{d} x \leq \frac{5}{a}|y|^{\alpha} \int_{B_{|y|-2}^{c}}\left(\varepsilon^{2}\left|\nabla u_{\varepsilon}\right|^{2}+V(x) u_{\varepsilon}^{2}\right) \mathrm{d} x .
$$

Next, we will estimate the second term on the right-hand side of (5.6). To this aim, it is useful to point out that Corollary 5.4 gives the validity of the main assumption (2.10) of Lemma 2.6; indeed, there exists $\varepsilon_{0} \in(0,1]$ and $M>0$ such that

$$
\left\|u_{\varepsilon}\right\|_{\varepsilon}^{2} \leq M \varepsilon^{2} \quad \forall \varepsilon \in\left(0, \varepsilon_{0}\right)
$$

From (3.1), for any $\gamma>0$, we get

$$
g_{\varepsilon}^{2}(x) \leq C_{1} Q(x) u_{\varepsilon}^{2}+C_{2}(\gamma) Q(x)\left(\mathrm{e}^{\gamma u_{\varepsilon}^{2}}-1\right), \quad x \in \mathbb{R}^{2},
$$

and hence

$$
\begin{aligned}
\int_{B(y, 2)} g_{\varepsilon}^{2}(x) \mathrm{d} x & \leq C_{1} \int_{B_{|y|-2}^{c}} Q(x) u_{\varepsilon}^{2} \mathrm{~d} x+C_{2}(\gamma) \int_{B_{|y|-2}^{c}} Q(x)\left(\mathrm{e}^{\gamma u_{\varepsilon}^{2}}-1\right) \mathrm{d} x \\
& \leq 2 \frac{b}{a} C_{1} \int_{B_{|y|-2}^{c}} V(x) u_{\varepsilon}^{2} \mathrm{~d} x+C_{2}(\gamma) \int_{B_{|y|-2}^{c}} Q(x)\left(\mathrm{e}^{\gamma u_{\varepsilon}^{2}}-1\right) \mathrm{d} x,
\end{aligned}
$$

where in the last inequality we used $(V)$ and $(Q)$ to deduce that $Q / V \leq 2 b / a$ on $B_{1}^{c}$. To estimate the second term on the right-hand side of (5.8), we apply Lemma 2.6. This can be done, in view of (5.7), if we choose $\gamma \in(0, \bar{\gamma})$ and $R \geq \bar{R}$, with $\bar{\gamma}$ and $\bar{R}$ given by Lemma 2.6; since $\varepsilon_{0} \in(0,1]$, the choices of both $\gamma$ and $R_{0}$ are independent of $\varepsilon \in\left(0, \varepsilon_{0}\right)$. In this way, we get

$$
\int_{B(y, 2)} g_{\varepsilon}^{2}(x) \mathrm{d} x \leq C_{3} \int_{B_{|y|-2}^{c}}\left(\varepsilon^{2}\left|\nabla u_{\varepsilon}\right|^{2}+V(x) u_{\varepsilon}^{2}\right) \mathrm{d} x,
$$

with $C_{3}>0$ independent of $\varepsilon \in\left(0, \varepsilon_{0}\right)$ and $y$, provided $|y|>2 R+2$.

What we proved so far shows the existence of $R_{0}>1$ such that, for any $\varepsilon \in\left(0, \varepsilon_{0}\right)$ and any $y \in \mathbb{R}^{2}$ with $|y|>2 R_{0}+2$, we have

$$
\left\|u_{\varepsilon}\right\|_{L^{\infty}(B(y, 1))} \leq C^{\prime \prime} \varepsilon^{-d}|y|^{\alpha / 2}\left[\int_{B_{|y|-2}^{c}}\left(\varepsilon^{2}\left|\nabla u_{\varepsilon}\right|^{2}+V(x) u_{\varepsilon}^{2}\right) \mathrm{d} x\right]^{1 / 2} .
$$


Finally, in view of (5.7), we can apply Lemma 4.2. Again, this can be done if we choose $R \geq R_{0}$, with $R_{0}$ given by Lemma 4.2 , and this choice is independent of $\varepsilon \in\left(0, \varepsilon_{0}\right)$. Since $|y|-2 \geq \frac{|y|}{2}$, we easily obtain (5.5) and the proof is complete.

Remark 5.6 Any weak solution $u_{\varepsilon}$ to $\left(\mathcal{P}_{\varepsilon}\right)$ satisfies $u_{\varepsilon} \in L^{\infty}\left(\mathbb{R}^{2}\right)$, for any $\varepsilon>0$. In fact, let $\varepsilon>0$ be fixed, and let $u_{\varepsilon}$ be any weak solution to $\left(\mathcal{P}_{\varepsilon}\right)$. Clearly, $u_{\varepsilon}$ satisfies (2.10), or equivalently (4.1), with $M=M(\varepsilon):=\left\|u_{\varepsilon}\right\|_{\varepsilon}^{2} / \varepsilon^{2}$. Therefore, we can argue as in the proof of Theorem 5.5 to conclude the validity of (5.5) with $C$ and $R$ depending on $\varepsilon$.

\subsection{Concentration behavior of mountain pass solutions}

The validity of the pointwise uniform exponential decay estimate expressed by Theorem 5.5 will play a crucial role, and from now on we will implicitly assume $\varepsilon>0$ to be sufficiently small.

Let $x_{\varepsilon} \in \mathbb{R}^{2}$ be any local maximum point of $u_{\varepsilon}$. As in [6, Lemmas 24 and 25], we show that $\left\{x_{\varepsilon}\right\}$ is bounded in $\mathbb{R}^{2}$ and $\left\|u_{\varepsilon}\right\|_{\infty} \geq c>0$. More precisely, we have the following result.

Lemma 5.7 There exist $c>0$ and $C>0$ such that for any local maximum point $x_{\varepsilon} \in \mathbb{R}^{2}$ of $u_{\varepsilon}$, it holds

(I) $u_{\varepsilon}\left(x_{\varepsilon}\right) \geq c$,

(II) $\left|x_{\varepsilon}\right| \leq C$.

Proof By contradiction, we assume that $u_{\varepsilon}\left(x_{\varepsilon}\right) \rightarrow 0$, for a sequence of local maximum points $x_{\varepsilon} \in \mathbb{R}^{2}$. Since $x_{\varepsilon}$ is a maximum point of $u_{\varepsilon}$, we have $\Delta u_{\varepsilon}\left(x_{\varepsilon}\right) \leq 0$, and hence

$$
V\left(x_{\varepsilon}\right) u_{\varepsilon}\left(x_{\varepsilon}\right) \leq-\Delta u_{\varepsilon}\left(x_{\varepsilon}\right)+V\left(x_{\varepsilon}\right) u_{\varepsilon}\left(x_{\varepsilon}\right)=Q\left(x_{\varepsilon}\right) f\left(u_{\varepsilon}\left(x_{\varepsilon}\right)\right) .
$$

Recalling that $u_{\varepsilon}>0$ and $\min _{x \in \mathbb{R}^{2}} \mathcal{A}(x)>0$, where $\mathcal{A}=V / Q$, we deduce that

$$
0<\min _{x \in \mathbb{R}^{2}} \mathcal{A}(x) \leq \frac{V\left(x_{\varepsilon}\right)}{Q\left(x_{\varepsilon}\right)} \leq \frac{f\left(u_{\varepsilon}\left(x_{\varepsilon}\right)\right)}{u_{\varepsilon}\left(x_{\varepsilon}\right)},
$$

which is in contradiction with condition $\left(f_{1}\right)$. Thus, (I) holds. To prove (II), it is enough to point out that if $\left|x_{\varepsilon}\right| \rightarrow+\infty$ then Theorem 5.5 yields $u_{\varepsilon}\left(x_{\varepsilon}\right) \rightarrow 0$, which contradicts (I).

In view of Lemma 5.7, given $\left\{x_{\varepsilon}\right\}$, where $x_{\varepsilon} \in \mathbb{R}^{2}$ is a local maximum point of $u_{\varepsilon}$, we have $x_{\varepsilon} \rightarrow x^{*}$ in $\mathbb{R}^{2}$ as $\varepsilon \rightarrow 0$, up to a subsequence. If we introduce the auxiliary function

$$
\psi_{\varepsilon}(x)=u_{\varepsilon}\left(\varepsilon x+x_{\varepsilon}\right), \quad x \in \mathbb{R}^{2},
$$

then $x=0$ is a local maximum point of $\psi_{\varepsilon}$. Moreover, $\psi_{\varepsilon}$ solves

$$
-\Delta \psi_{\varepsilon}(x)+V\left(\varepsilon x+x_{\varepsilon}\right) \psi_{\varepsilon}(x)=Q\left(\varepsilon x+x_{\varepsilon}\right) f\left(\psi_{\varepsilon}(x)\right), \quad x \in \mathbb{R}^{2},
$$

and we can compute

$$
\int_{\mathbb{R}^{2}}\left(\left|\nabla \psi_{\varepsilon}\right|^{2}+V\left(\varepsilon x+x_{\varepsilon}\right) \psi_{\varepsilon}^{2}\right) \mathrm{d} x=\varepsilon^{-2}\left\|u_{\varepsilon}\right\|_{\varepsilon}^{2} .
$$

Using again condition $(V)$ and the boundedness of $\left\{x_{\varepsilon}\right\}$ expressed by Lemma 5.7(II), we have the existence of a constant $\tilde{a}>0$ such that

$$
\int_{\mathbb{R}^{2}}\left(\left|\nabla \psi_{\varepsilon}\right|^{2}+\frac{\tilde{a}}{1+|x|^{\alpha}} \psi_{\varepsilon}^{2}(x)\right) \mathrm{d} x \leq \varepsilon^{-2}\left\|u_{\varepsilon}\right\|_{\varepsilon}^{2} \leq M,
$$


where we also applied Corollary 5.4. Therefore, by Schauder estimates, $\left\{\psi_{\varepsilon}\right\}$ is bounded in $\mathcal{C}_{\text {loc }}^{2}\left(\mathbb{R}^{2}\right)$, and hence $\psi_{\varepsilon} \rightarrow U^{*}$ in $\mathcal{C}_{\text {loc }}^{2}\left(\mathbb{R}^{2}\right)$ as $\varepsilon \rightarrow 0$. Passing to the limit in (5.9), we deduce that $U^{*}$ is a classical solution to the limiting problem (5.1) with $\xi=x^{*}$, i.e.,

$$
-\Delta U^{*}+V\left(x^{*}\right) U^{*}=Q\left(x^{*}\right) f\left(U^{*}\right) \text { in } \mathbb{R}^{2} .
$$

Arguing as in Theorem 5.5, we deduce that $\psi_{\varepsilon} \rightarrow U^{*}$ in $L^{\infty}\left(\mathbb{R}^{2}\right)$ as $\varepsilon \rightarrow 0$. The positivity of $\psi_{\varepsilon}$ yields $U^{*} \geq 0$, and since $x=0$ is a maximum point of $\psi_{\varepsilon}$ then $x=0$ is also a maximum point of $U^{*}$. Moreover, $\psi_{\varepsilon}(0)=u_{\varepsilon}\left(x_{\varepsilon}\right)$ and, as a consequence of Lemma 5.7(I), $U^{*}(0) \neq 0$ which yields $U^{*} \not \equiv 0$. It follows from the maximum principle that $U^{*}>0$. Because $\psi_{\varepsilon} \rightarrow U^{*}$ in $\mathcal{C}_{\text {loc }}^{2}\left(\mathbb{R}^{2}\right)$, for any $R>0$, we have

$$
\begin{aligned}
\int_{B_{R}}\left(\left|\nabla U^{*}\right|^{2}+V\left(x^{*}\right)\left(U^{*}\right)^{2}\right) \mathrm{d} x & =\lim _{\varepsilon \rightarrow 0} \int_{B_{R}}\left(\left|\nabla \psi_{\varepsilon}\right|^{2}+V\left(\varepsilon x+x_{\varepsilon}\right) \psi_{\varepsilon}^{2}\right) \mathrm{d} x \\
& \leq \limsup _{\varepsilon \rightarrow 0} \varepsilon^{-2}\left\|u_{\varepsilon}\right\|_{\varepsilon}^{2} \leq M,
\end{aligned}
$$

and hence $U^{*} \in H^{1}\left(\mathbb{R}^{2}\right)$.

Next, we consider the energy functional $L_{x^{*}}: H^{1}\left(\mathbb{R}^{2}\right) \rightarrow \mathbb{R}$ defined in (5.2) with $\xi=x^{*}$.

Lemma 5.8 The following inequality holds

$$
L_{x^{*}}\left(U^{*}\right) \leq \liminf _{\varepsilon \rightarrow 0} \varepsilon^{-2} I_{\varepsilon}\left(u_{\varepsilon}\right)=\liminf _{\varepsilon \rightarrow 0} \varepsilon^{-2} c_{\varepsilon} .
$$

Proof We have

$$
\varepsilon^{-2} c_{\varepsilon}=\varepsilon^{-2} I_{\varepsilon}\left(u_{\varepsilon}\right)=\frac{1}{2} \int_{\mathbb{R}^{2}}\left(\left|\nabla \psi_{\varepsilon}\right|^{2}+V\left(\varepsilon x+x_{\varepsilon}\right) \psi_{\varepsilon}^{2}\right) \mathrm{d} x-\int_{\mathbb{R}^{2}} Q\left(\varepsilon x+x_{\varepsilon}\right) F\left(\psi_{\varepsilon}\right) \mathrm{d} x,
$$

and we define

$$
h_{\varepsilon}(x)=\frac{1}{2}\left(\left|\nabla \psi_{\varepsilon}\right|^{2}+V\left(\varepsilon x+x_{\varepsilon}\right) \psi_{\varepsilon}^{2}\right)-Q\left(\varepsilon x+x_{\varepsilon}\right) F\left(\psi_{\varepsilon}\right),
$$

so that

$$
\varepsilon^{-2} c_{\varepsilon}=\int_{\mathbb{R}^{2}} h_{\varepsilon}(x) \mathrm{d} x
$$

For any $R>0$,

$$
\lim _{\varepsilon \rightarrow 0} \int_{B_{R}} h_{\varepsilon}(x) \mathrm{d} x=\frac{1}{2} \int_{B_{R}}\left(\left|\nabla U^{*}\right|^{2}+V\left(x^{*}\right)\left(U^{*}\right)^{2}\right) \mathrm{d} x-\int_{B_{R}} Q\left(x^{*}\right) F\left(U^{*}\right) \mathrm{d} x .
$$

Since $U^{*} \in H^{1}\left(\mathbb{R}^{2}\right)$, for any $v>0$ there exists $R_{v}>0$ such that

$$
\int_{B_{R}^{c}}\left(\left|\nabla U^{*}\right|^{2}+V\left(x^{*}\right)\left(U^{*}\right)^{2}\right) \mathrm{d} x<v, \text { for any } R \geq R_{v},
$$

and hence

$$
\begin{aligned}
\lim _{\varepsilon \rightarrow 0} \int_{B_{R}} h_{\varepsilon}(x) \mathrm{d} x & \geq \frac{1}{2} \int_{\mathbb{R}^{2}}\left(\left|\nabla U^{*}\right|^{2}+V\left(x^{*}\right)\left(U^{*}\right)^{2}\right) \mathrm{d} x-\int_{\mathbb{R}^{2}} Q\left(x^{*}\right) F\left(U^{*}\right) \mathrm{d} x-\frac{v}{2} \\
& =L_{x^{*}}\left(U^{*}\right)-\frac{v}{2}
\end{aligned}
$$


In view of (5.12), the proof is complete if we show that, for some $R \geq R_{v}$,

$$
\liminf _{\varepsilon \rightarrow 0} \int_{B_{R}^{c}} h_{\varepsilon}(x) \mathrm{d} x \geq \frac{-v}{2} .
$$

To this aim, we consider $\eta \in \mathcal{C}^{\infty}\left(\mathbb{R}^{2},[0,1]\right)$ satisfying

$$
\eta \equiv 0 \text { in } B_{R-1}, \quad \eta \equiv 1 \text { in } B_{R}^{c}, \quad \text { and }|\nabla \eta(x)| \leq 2 \forall x \in \mathbb{R}^{2} .
$$

Using $\eta \psi_{\varepsilon}$ as a test function in equation (5.9), by $\left(f_{2}\right)$ we obtain

$$
\begin{aligned}
0= & \int_{B_{R}^{c}}\left(\left|\nabla \psi_{\varepsilon}\right|^{2}+V\left(\varepsilon x+x_{\varepsilon}\right) \psi_{\varepsilon}^{2}\right) \mathrm{d} x-\int_{B_{R}^{c}} Q\left(\varepsilon x+x_{\varepsilon}\right) f\left(\psi_{\varepsilon}\right) \psi_{\varepsilon} \mathrm{d} x \\
& +\int_{B_{R} \backslash B_{R-1}}\left[\left|\nabla \psi_{\varepsilon}\right|^{2} \eta+\psi_{\varepsilon} \nabla \eta \nabla \psi_{\varepsilon}+V\left(\varepsilon x+x_{\varepsilon}\right) \eta \psi_{\varepsilon}^{2}-Q\left(\varepsilon x+x_{\varepsilon}\right) f\left(\psi_{\varepsilon}\right) \eta \psi_{\varepsilon}\right] \mathrm{d} x \\
= & 2 \int_{B_{R}^{c}} h_{\varepsilon} \mathrm{d} x-\int_{B_{R}^{c}} Q\left(\varepsilon x+x_{\varepsilon}\right)\left[f\left(\psi_{\varepsilon}\right) \psi_{\varepsilon}-2 F\left(\psi_{\varepsilon}\right)\right] \mathrm{d} x+E_{\varepsilon} \leq 2 \int_{B_{R}^{c}} h_{\varepsilon} \mathrm{d} x+E_{\varepsilon}
\end{aligned}
$$

where

$$
E_{\varepsilon}:=\int_{B_{R} \backslash B_{R-1}}\left[\left|\nabla \psi_{\varepsilon}\right|^{2} \eta+\psi_{\varepsilon} \nabla \eta \nabla \psi_{\varepsilon}+V\left(\varepsilon x+x_{\varepsilon}\right) \eta \psi_{\varepsilon}^{2}-Q\left(\varepsilon x+x_{\varepsilon}\right) f\left(\psi_{\varepsilon}\right) \eta \psi_{\varepsilon}\right] \mathrm{d} x .
$$

The convergence $\psi_{\varepsilon} \rightarrow U^{*}$ in $B_{R} \backslash B_{R-1}$, as $\varepsilon \rightarrow 0$, implies $\lim _{\sup } \rightarrow 0\left|E_{\varepsilon}\right| \leq v$ for $R \geq R_{\nu}$ sufficiently large, and hence

$$
\liminf _{\varepsilon \rightarrow 0} \int_{B_{R}^{c}} h_{\varepsilon}(x) \mathrm{d} x \geq \liminf _{\varepsilon \rightarrow 0} \frac{-E_{\varepsilon}}{2} \geq \frac{-v}{2} .
$$

Lemma 5.9 The limit point $x^{*}$ is a global minimum point of the auxiliary potential $\mathcal{A}=V / Q$ and $U^{*}$ is a ground-state solution of the limiting problem (5.10).

Proof Let $\xi \in \mathbb{R}^{2}$ and let $m_{\xi}$ be the least energy level of the functional $L_{\xi}$ introduced in (5.2). By contradiction, we assume the existence of $\xi^{*} \in \mathbb{R}^{2}$ such that $\mathcal{A}\left(\xi^{*}\right)<\mathcal{A}\left(x^{*}\right)$. Recalling (5.4), then we have $m_{\xi^{*}}<m_{x^{*}}$. On the other hand, combining Proposition 5.3 and Lemma 5.8 , we get

$$
m_{x^{*}} \leq L_{x^{*}}\left(U^{*}\right) \leq \liminf _{\varepsilon \rightarrow 0} \varepsilon^{-2} I_{\varepsilon}\left(u_{\varepsilon}\right) \leq m_{\xi}, \quad \forall \xi \in \mathbb{R}^{2} .
$$

Therefore, $x^{*}$ must be a global minimum point of $\mathcal{A}$. Moreover, $m_{x^{*}}=L_{x^{*}}\left(U^{*}\right)$, and $U^{*}$ is a ground-state solution of (5.10).

Lemma 5.10 If $x_{\varepsilon}$ and $y_{\varepsilon}$ are maximum points of $u_{\varepsilon}$ then $\left|x_{\varepsilon}-y_{\varepsilon}\right| \leq C \varepsilon$. If in addition $f$ is increasing on $(0,+\infty)$, then $u_{\varepsilon}$ has a unique maximum point in $\mathbb{R}^{2}$.

Proof Let $x_{\varepsilon}$ and $y_{\varepsilon}$ be maximum points of $u_{\varepsilon}$. In view of Lemma 5.7(II), without loss of generality, we can assume that $x_{\varepsilon} \rightarrow x^{*}$ and $y_{\varepsilon} \rightarrow y^{*}$, in $\mathbb{R}^{2}$. We proved that the rescaled functions $\psi_{\varepsilon}(x):=u_{\varepsilon}\left(\varepsilon x+x_{\varepsilon}\right)$ and $\tilde{\psi}_{\varepsilon}(x):=u_{\varepsilon}\left(\varepsilon x+y_{\varepsilon}\right)$ satisfy $\psi_{\varepsilon} \rightarrow U^{*}$ and $\tilde{\psi}_{\varepsilon} \rightarrow \tilde{U}^{*}$ in $C_{\mathrm{loc}}^{2}\left(\mathbb{R}^{2}\right)$, where $U^{*}$ and $\tilde{U}^{*}$ are positive ground-state solutions of the limiting problem (5.1) with $\xi=x^{*}$ and $\xi=y^{*}$, respectively. We also pointed out that $x=0$ is a maximum point of both $U^{*}$ and $\tilde{U}^{*}$. Let $z_{\varepsilon}:=\varepsilon^{-1}\left(y_{\varepsilon}-x_{\varepsilon}\right)$. Clearly, we have the following alternative:

(i) either $\left\{z_{\varepsilon}\right\}$ is bounded, and up to a subsequence, $z_{\varepsilon} \rightarrow z^{*}$ in $\mathbb{R}^{2}$; 
(ii) or $\left\{z_{\varepsilon}\right\}$ is unbounded, and up to a subsequence, $\left|z_{\varepsilon}\right| \rightarrow \infty$.

The first part of the statement of the lemma is equivalent to show that (ii) cannot occur. Assume by contradiction that $\left|z_{\varepsilon}\right| \rightarrow \infty$. For any $R>0$, the balls $B_{R}$ and $B\left(z_{\varepsilon}, R\right)$ are disjoint, provided $\varepsilon>0$ is sufficiently small. Let $h_{\varepsilon}$ be the function defined by (5.11) in terms of $\psi_{\varepsilon}$. In particular (5.13) holds, i.e., for any $v>0$ there exits $R=R(v)>0$ such that

$$
\lim _{\varepsilon \rightarrow 0} \int_{B_{R}} h_{\varepsilon}(x) \mathrm{d} x \geq L_{x^{*}}\left(U^{*}\right)-\frac{v}{2},
$$

and arguing as in the proof of Lemma 5.8 (after a suitable change of variable), we get also $\lim _{\varepsilon \rightarrow 0} \int_{B\left(z_{\varepsilon}, R\right)} h_{\varepsilon}(x) \mathrm{d} x \geq L_{y^{*}}\left(\tilde{U}^{*}\right)-\frac{v}{2}, \quad$ along with $\liminf _{\varepsilon \rightarrow 0} \int_{\left(B\left(z_{\varepsilon}, R\right) \cup B_{R}\right)^{c}} h_{\varepsilon}(x) \mathrm{d} x \geq-v$. Therefore,

$$
\liminf _{\varepsilon \rightarrow 0} \int_{\mathbb{R}^{2}} h_{\varepsilon}(x) \mathrm{d} x \geq L_{x^{*}}\left(U^{*}\right)+L_{y^{*}}\left(\tilde{U}^{*}\right)-2 v,
$$

and combining (5.12) with the fact that $v>0$ is arbitrary, we get

$$
\liminf _{\varepsilon \rightarrow 0} \varepsilon^{-2} c_{\varepsilon}=\liminf _{\varepsilon \rightarrow 0} \int_{\mathbb{R}^{2}} h_{\varepsilon}(x) \mathrm{d} x \geq L_{x^{*}}\left(U^{*}\right)+L_{y^{*}}\left(\tilde{U}^{*}\right) .
$$

Proposition 5.3 yields $\lim \inf _{\varepsilon \rightarrow 0} \varepsilon^{-2} c_{\varepsilon} \leq m_{\xi}$, for all $\xi \in \mathbb{R}^{2}$. Since $x^{*}$ and $y^{*}$ are global minimum points of $\mathcal{A}$, we have $\mathcal{A}\left(x^{*}\right)=\mathcal{A}\left(y^{*}\right)$ and $m_{x^{*}}=m_{y^{*}}$ (see Remark 5.2). Hence

$$
L_{x^{*}}\left(U^{*}\right)+L_{y^{*}}\left(\tilde{U}^{*}\right) \leq \frac{1}{2}\left(m_{x^{*}}+m_{y^{*}}\right)=\frac{1}{2}\left(L_{x^{*}}\left(U^{*}\right)+L_{y^{*}}\left(\tilde{U}^{*}\right)\right),
$$

which is impossible, and this rules out (ii).

From now on, we furthermore assume that $f$ is increasing on $(0,+\infty)$, and we aim to show that $x_{\varepsilon}=y_{\varepsilon}$. To this aim, it is enough to prove that (i) cannot occur unless $x_{\varepsilon}=y_{\varepsilon}$.

Since $U^{*}$ is a ground-state solution to the limiting problem (5.1) with $\xi=x^{*}$, it is well known that, for some $x_{0} \in \mathbb{R}^{2}, U^{*}\left(\cdot+x_{0}\right)$ is radially symmetric and strictly decreasing (see [8] and [7]). Since $x=0$ is a maximum point of $U^{*}$, we deduce that $x_{0}=0$. Using the symmetry, positivity and monotonicity properties of $U^{*}$ together with the monotonicity of $f$, we can easily show that $\Delta U^{*}(0)<0$. In particular, $U_{r r}^{*}(0)<0$, with $r=|x|$, and in view of the convergence $\psi_{\varepsilon} \rightarrow U^{*}$ in $C_{\text {loc }}^{2}\left(\mathbb{R}^{2}\right)$, we can apply [29, Lemma 4.2] to get the existence of $R>0$ such that, for any small $\varepsilon>0, x=0$ is the unique critical point of $\psi_{\varepsilon}$ in $B_{R}$.

If (i) occurs, since $z_{\varepsilon}$ is a maximum point of $\psi_{\varepsilon}$, then $z^{*}$ is a maximum point of $U^{*}$, and hence $z^{*}=0$. Thus, $z_{\varepsilon} \in B_{R}$ for any small $\varepsilon>0$, and the choice of $R$ implies $z_{\varepsilon}=0$, equivalently $x_{\varepsilon}=y_{\varepsilon}$. Therefore, for small $\varepsilon>0, u_{\varepsilon}$ has a unique local (hence global) maximum point in $\mathbb{R}^{2}$.

Remark 5.11 The monotonicity assumption on $f$ in Lemma 5.10 is required to guarantee that $U_{r r}^{*}(0)<0$. The same conclusion can be reached, by means of a classical fixed point argument, if $f$ is locally Lipschitz continuous. 


\section{Critical case-existence}

This section is devoted to the proof of Theorem 1.4. More precisely, for any fixed $\varepsilon>0$, we prove the existence of a mountain pass solution to $\left(\mathcal{P}_{\varepsilon}\right)$ under the assumptions:

- $(V)$ and $(Q)$ hold with $\alpha$ and $\beta$ in the range (1.1),

- $f: \mathbb{R} \rightarrow \mathbb{R}$ is a continuous function, with critical exponential growth $\left(f_{0}^{\prime}\right)$, satisfying $\left(f_{1}\right),\left(f_{2}\right),\left(f_{3}\right)$ and $\left(f_{4}^{\prime}\right)$.

The proof follows the same lines of [21, Section 5], with obvious modifications due to the presence of the parameter $\varepsilon>0$, and it is based on the classical mountain pass theorem. Therefore, we will only sketch the main steps needed to ensure that the mountain pass theorem can actually be applied to obtain a nontrivial mountain pass solution. For the proof of the fact that mountain pass solutions belong to $L^{2}\left(\mathbb{R}^{2}\right)$, we refer the reader to [21, Proposition 2.2].

Let

$$
I_{\varepsilon}(u):=\frac{1}{2}\|u\|_{\varepsilon}^{2}-\int_{\mathbb{R}^{2}} Q(x) F(u) \mathrm{d} x \quad \forall u \in \mathcal{H}_{\varepsilon},
$$

and

$c_{\varepsilon}:=\inf _{\gamma \in \Gamma_{\varepsilon}} \sup _{t \in[0,1]} I_{\varepsilon}(\gamma(t)), \quad$ where $\Gamma_{\varepsilon}:=\left\{\gamma \in \mathcal{C}\left([0,1], \mathcal{H}_{\varepsilon}\right) \mid \gamma(0)=0\right.$ and $\left.I_{\varepsilon}(\gamma(1))<0\right\}$.

Step $1 I_{\varepsilon} \in \mathcal{C}^{1}\left(\mathcal{H}_{\varepsilon}, \mathbb{R}\right), I_{\varepsilon}$ has a mountain pass geometry on $\mathcal{H}_{\varepsilon}$, and hence the mountain pass level $c_{\varepsilon}$ is well defined and positive.

In view of $\left(f_{0}^{\prime}\right)$ and $\left(f_{1}\right)$, we have the validity of (3.1) and (3.2) for any $\gamma>\gamma_{0}, q \geq 0$ and $\delta>0$ (here, $\gamma_{0}$ is given by $\left(f_{0}^{\prime}\right)$ and determines the critical growth of $f$ at infinity). This is enough to deduce, as in Sect. 6, that $I_{\varepsilon}$ is a $\mathcal{C}^{1}$-functional on $\mathcal{H}_{\varepsilon}$. Moreover, the conclusion of Lemma 3.1 still holds (see [21, Lemma 5.1]).

Step 2 We have

$$
c_{\varepsilon}<2 \pi \varepsilon^{2} / \gamma_{0},
$$

where $\gamma_{0}$ is given by condition $\left(f_{0}^{\prime}\right)$.

In view of $\left(f_{4}^{\prime}\right)$, the proof of [21, Lemma 5.2] can be repeated using the same classical Moser's sequence of functions and taking into account the presence of the parameter $\varepsilon>0$. Arguing in this way, we deduce (6.1).

Step 3 Let $c \in \mathbb{R}$, and let $\left\{u_{n}\right\}_{n} \subset \mathcal{H}_{\varepsilon}$ be any $(P S)_{c}$-sequence for $I_{\mathcal{E}}$. Then $\left\{u_{n}\right\}_{n}$ is bounded in $\mathcal{H}_{\varepsilon}$, and

$$
\sup _{n} \int_{\mathbb{R}^{2}} Q(x) f\left(u_{n}\right) u_{n} \mathrm{~d} x<+\infty .
$$

Moreover, if $u_{n} \rightarrow u_{\varepsilon}$ in $\mathcal{H}_{\varepsilon}$ then

$$
\lim _{n \rightarrow+\infty} \int_{\mathbb{R}^{2}} Q(x) F\left(u_{n}\right) \mathrm{d} x=\int_{\mathbb{R}^{2}} Q(x) F\left(u_{\varepsilon}\right) \mathrm{d} x .
$$

In view of $\left(f_{2}\right)$, the proof of the boundedness of $\left\{u_{n}\right\}_{n}$ and the proof of (6.2) are standard and can be carried out as in [21, Lemma 5.3]. Moreover, using $\left(f_{3}\right)$ and the compactness of the embedding $\mathcal{H}_{\varepsilon} \hookrightarrow L_{Q}^{2}\left(\mathbb{R}^{2}\right)$ expressed by Theorem $2.5,(6.3)$ can be deduced as in [21, Lemma 5.4].

Step $4 I_{\varepsilon}$ satisfies the $(P S)_{c_{\varepsilon}}$-condition at the mountain pass level $c_{\varepsilon}$.

We follow closely the arguments introduced in [16]. Let $\left\{u_{n}\right\}_{n}$ be a $(P S)_{c_{\varepsilon}}$-sequence for $I_{\varepsilon}$, i.e.,

$$
\lim _{n \rightarrow+\infty} I_{\varepsilon}\left(u_{n}\right)=c_{\varepsilon}
$$


and

$$
\left|\left\langle u_{n}, v\right\rangle_{\varepsilon}-\int_{\mathbb{R}^{2}} Q(x) f\left(u_{n}\right) v \mathrm{~d} x\right| \leq \delta_{n}\|v\|_{\varepsilon} \quad \forall v \in \mathcal{H}_{\varepsilon}, \text { where } \lim _{n \rightarrow+\infty} \delta_{n}=0 .
$$

In view of the boundedness of $\left\{u_{n}\right\}_{n}$ in $\mathcal{H}_{\varepsilon}$, up to a subsequence, $u_{n} \rightarrow u_{\varepsilon}$ in $\mathcal{H}_{\varepsilon}$.

First, we prove that $u_{\varepsilon} \not \equiv 0$. If not then $u_{\varepsilon} \equiv 0$, and combining (6.4) with (6.3), we get

$$
\lim _{n \rightarrow \infty}\left\|u_{n}\right\|_{\varepsilon}^{2}=\lim _{n \rightarrow \infty} 2\left[c_{\varepsilon}+\int_{\mathbb{R}^{2}} Q(x) F\left(u_{n}\right) \mathrm{d} x\right]=2 c_{\varepsilon}>0 .
$$

On the other hand, using the compact embeddings expressed by Theorem 2.5 and arguing as in [21, Section 5.3], we deduce that $u_{n} \rightarrow 0$ strongly in $\mathcal{H}_{\varepsilon}$, and we reach a contradiction.

Clearly, $I_{\varepsilon}\left(u_{\varepsilon}\right) \leq c_{\varepsilon}$. Our aim is to show the strong convergence $u_{n} \rightarrow u_{\varepsilon}$ in $\mathcal{H}_{\varepsilon}$, and this is equivalent to $I_{\varepsilon}\left(u_{\varepsilon}\right)=c_{\varepsilon}$. We will prove that if

$$
I_{\varepsilon}\left(u_{\varepsilon}\right)<c_{\varepsilon}
$$

then

$$
\lim _{n \rightarrow+\infty} \int_{\mathbb{R}^{2}} Q(x) f\left(u_{n}\right)\left(u_{n}-u_{\varepsilon}\right) \mathrm{d} x=0,
$$

and hence (6.6) cannot happen. Indeed, (6.5) and (6.7) yield $\left\langle u_{n}, u_{n}-u_{\varepsilon}\right\rangle_{\varepsilon} \rightarrow 0$, and we deduce that $u_{n} \rightarrow u_{\varepsilon}$ strongly in $\mathcal{H}_{\varepsilon}$ which contradicts (6.6).

To prove (6.7), let $\gamma>\gamma_{0}$, so that (3.1) holds with $q \geq 0$ and $\delta>0$. Using (3.1) with $q=0$, using the continuity of the embedding $\mathcal{H}_{\varepsilon} \hookrightarrow L_{Q}^{2}\left(\mathbb{R}^{2}\right)$ expressed by Theorem 2.5 , and the boundedness of $\left\{u_{n}\right\}_{n}$ in $\mathcal{H}_{\varepsilon}$, for any $\delta>0$ we have

$$
\begin{aligned}
& \left|\int_{\mathbb{R}^{2}} Q(x) f\left(u_{n}\right)\left(u_{n}-u_{\varepsilon}\right) \mathrm{d} x\right| \leq C_{1} \delta+C_{2}(\gamma, \delta) \int_{\mathbb{R}^{2}} Q(x)\left|u_{n}-u_{\varepsilon}\right|\left(\mathrm{e}^{\gamma u_{n}^{2}}-1\right) \mathrm{d} x \\
& \leq C_{1} \delta+C_{2}(\gamma, \delta)\left(\int_{\mathbb{R}^{2}} Q(x)\left|u_{n}-u_{\varepsilon}\right|^{p^{\prime}} \mathrm{d} x\right)^{\frac{1}{p^{\prime}}}\left(\int_{\mathbb{R}^{2}} Q(x)\left(\mathrm{e}^{\gamma p u_{n}^{2}}-1\right) \mathrm{d} x\right)^{\frac{1}{p}},
\end{aligned}
$$

where in the last estimate we applied Hölder's inequality with $p>1$ and $\frac{1}{p}+\frac{1}{p^{\prime}}=1$. Note that, Theorem 2.5 yields the compactness of the embedding $\mathcal{H}_{\varepsilon} \hookrightarrow L_{Q}^{p^{\prime}}\left(\mathbb{R}^{2}\right)$ for any $p^{\prime} \geq 2$. Therefore, if we assume the existence of $\gamma>\gamma_{0}$ and $p \in(1,2]$, such that

$$
\sup _{n} E_{n}(\gamma, p)<\infty, \quad \text { where } E_{n}(\gamma, p):=\int_{\mathbb{R}^{2}} Q(x)\left(\mathrm{e}^{\gamma p u_{n}^{2}}-1\right) \mathrm{d} x
$$

then

$$
\left|\int_{\mathbb{R}^{2}} Q(x) f\left(u_{n}\right)\left(u_{n}-u_{\varepsilon}\right) \mathrm{d} x\right| \leq C_{1} \delta+C_{2}(\gamma, \delta)\left(\sup _{n} E_{n}(\gamma, p)\right)^{\frac{1}{p}}\left(\int_{\mathbb{R}^{2}} Q(x)\left|u_{n}-u_{\varepsilon}\right|^{p^{\prime}} \mathrm{d} x\right)^{\frac{1}{p^{\prime}}},
$$

and the proof of (6.7) is complete.

We obtain (6.8) as a consequence of the following Lions-type concentration-compactness lemma in $\mathcal{H}_{\varepsilon}$.

Lemma 6.1 Suppose that $(V)$ and $(Q)$ hold with $\alpha \in(0,2)$ and $\beta \in[\alpha,+\infty)$, respectively, and let $\varepsilon>0$. If $\left\{v_{n}\right\}_{n} \subset \mathcal{H}_{\varepsilon}$ satisfies $\left\|v_{n}\right\|_{\varepsilon}=\varepsilon$ for any $n \geq 1$, and $v_{n} \rightarrow v_{\varepsilon}$ in $\mathcal{H}_{\varepsilon}$ with $\left\|v_{\varepsilon}\right\|_{\varepsilon}<\varepsilon$ then

$$
\sup _{n} \int_{\mathbb{R}^{2}} Q(x)\left(\mathrm{e}^{q v_{n}^{2}}-1\right) \mathrm{d} x<+\infty \quad \forall q \in\left(0, \frac{\varepsilon^{2}}{\varepsilon^{2}-\left\|v_{\varepsilon}\right\|_{\varepsilon}^{2}} 4 \pi\right) .
$$


Proof In view of the subcritical Trudinger-Moser inequality expressed by Theorem 1.1, and recalling that $\mathcal{H}_{\varepsilon}$ is a Hilbert space, the proof of (6.9) follows the same lines of [19, Lemma 2.6].

We apply the above lemma to

$$
v_{\varepsilon}:=\varepsilon u_{\varepsilon}\left[2\left(c_{\varepsilon}+\int_{\mathbb{R}^{2}} Q(x) F\left(u_{\varepsilon}\right) \mathrm{d} x\right)\right]^{-\frac{1}{2}} \quad \text { and } \quad v_{n}:=\frac{\varepsilon u_{n}}{\left\|u_{n}\right\|_{\varepsilon}} .
$$

Indeed, $\left\|v_{n}\right\|_{\varepsilon}=\varepsilon$ and $v_{n} \rightarrow v_{\varepsilon}$ in $\mathcal{H}_{\varepsilon}$. Moreover, using (6.6), it is easy to see that $\left\|v_{\varepsilon}\right\|_{\varepsilon}<\varepsilon$, and hence (6.9) holds. Note that, since $v_{\varepsilon} \not \equiv 0,(6.9)$ improves the Trudinger-Moser inequality (1.3) along the sequence $\left\{v_{n}\right\}_{n}$, and we can rewrite

$$
\frac{\varepsilon^{2}}{\varepsilon^{2}-\left\|v_{\varepsilon}\right\|_{\varepsilon}^{2}} 4 \pi=\frac{2 \pi}{c_{\varepsilon}-I_{\varepsilon}\left(u_{\varepsilon}\right)} \lim _{n \rightarrow+\infty}\left\|u_{n}\right\|_{\varepsilon}^{2} .
$$

Clearly

$$
\sup _{n} E_{n}(\gamma, p)=\sup _{n} \int_{\mathbb{R}^{2}} Q(x)\left(\mathrm{e}^{\gamma p \frac{\left\|u_{n}\right\|_{\varepsilon}^{2}}{\varepsilon^{2}} v_{n}^{2}}-1\right) \mathrm{d} x<+\infty,
$$

provided the following inequality holds

$$
\gamma p\left\|u_{n}\right\|_{\varepsilon}^{2}<\varepsilon^{2} \frac{2 \pi}{c_{\varepsilon}-I_{\varepsilon}\left(u_{\varepsilon}\right)} \lim _{n \rightarrow+\infty}\left\|u_{n}\right\|_{\varepsilon}^{2},
$$

at least for any $n$ sufficiently large. This is actually the case in view of the fact that $c_{\varepsilon}<$ $2 \pi \varepsilon^{2} / \gamma_{0}$ and $I_{\varepsilon}\left(u_{\varepsilon}\right) \geq 0$, equivalently

$$
\varepsilon^{2} \frac{2 \pi}{c_{\varepsilon}-I_{\varepsilon}\left(u_{\varepsilon}\right)}>\gamma_{0},
$$

and hence (6.10) holds for any $n$ large if we choose $\gamma>\gamma_{0}$ sufficiently close to $\gamma_{0}$ and $p \in(1,2]$ sufficiently close to 1 . With this remark, the proof of $(6.8)$ is complete.

Acknowledgements J.M. do Ó and E. Gloss were partially supported by The National Institute of Science and Technology of Mathematics ICNT-Mat, CAPES and CNPq/Brazil. F. Sani is a member of GNAMPA/INdAM, and she was partially supported by the FFABR grant of the Italian Ministry of Education, Universities and Research. This work was done while E. Gloss was visiting the University of Milano.

\section{References}

1. Adachi, S., Tanaka, K.: Trudinger type inequalities in $\mathbb{R}^{N}$ and their best exponents. Proc. Am. Math. Soc. 128, 2051-2057 (2000)

2. Adimurthi: Existence of positive solutions of the semilinear Dirichlet problem with critical growth for the $n$-Laplacian. Ann. Scuola Norm. Sup. Pisa Cl. Sci. 17(4), 393-413 (1990)

3. Adimurthi, Yang, Y.: An interpolation of Hardy inequality and Trudinger-Moser inequality in $\mathbb{R}^{N}$ and its applications. Int. Math. Res. Not. IMRN 13, 2394-2426 (2010)

4. Alves, C.O., de Freitas, L.R.: Multiplicity results for a class of quasilinear equations with exponential critical growth. Math. Nachr. 291, 222-224 (2018)

5. Alves, C.O., Figueiredo, G.M.: On multiplicity and concentration of positive solutions for a class of quasilinear problems with critical exponential growth in $\mathbb{R}^{N}$. J. Differ. Equ. 246, 1288-1311 (2009)

6. Ambrosetti, A., Felli, V., Malchiodi, A.: Ground states of nonlinear Schrödinger equations with potentials vanishing at infinity. J. Eur. Math. Soc. (JEMS) 7, 117-144 (2005)

7. Berestycki, H., Gallouët, T., Kavian, O.: Équations de champs scalaires euclidiens non linéaires dans le plan. C. R. Acad. Sci. Paris Sér. I Math. 297, 307-310 (1983) 
8. Byeon, J., Jeanjean, L., Maris, M.: Symmetry and monotonicity of least energy solutions. Calc. Var. Partial Differ. Equ. 36, 481-492 (2009)

9. Byeon, J., Jeanjean, L., Tanaka, K.: Standing waves for nonlinear Schrödinger equations with a general nonlinearity: one and two dimensional cases. Commun. Partial Differ. Equ. 33, 1113-1136 (2008)

10. Bonheure, D., di Cosmo, J., Van Schaftingen, J.: Nonlinear Schrödinger equation with unbounded or vanishing potentials: solutions concentrating on lower dimensional spheres. J. Differ. Equ. 252, 941-968 (2012)

11. Bonheure, D., Van Schaftingen, J.: Bound state solutions for a class of nonlinear Schrödinger equations. Rev. Mat. Iberoam. 24, 297-351 (2008)

12. Cao, D., Peng, S.: Semi-classical bound states for Schrödinger equations with potentials vanishing or unbounded at infinity. Commun. Partial Differ. Equ. 34, 1566-1591 (2009)

13. Cassani, D., do Ó, J.M., Zhang, J.: Multi-bump solutions for singularly perturbed Schrödinger equations in R2 with general nonlinearities. Topol. Methods Nonlinear Anal. 49, 205-231 (2017)

14. Cassani, D., Sani, F., Tarsi, C.: Equivalent Moser type inequalities in $\mathbb{R}^{2}$ and the zero mass case. J. Funct. Anal. 267, 4236-4263 (2014)

15. Cui, D.C., Zhang, J.H., Fei, M.W.: Existence and concentration of bound states of a class of nonlinear Schrödinger equations in $\mathbb{R}^{N}$ with potential tending to zero at infinity. Acta Math. Sin. (Engl. Ser.) 28, 2243-2274 (2012)

16. de Figueiredo, D.G., Miyagaki, O.H., Ruf, B.: Elliptic equations in $\mathbb{R}^{2}$ with nonlinearities in the critical growth range. Calc. Var. Partial Differ. Equ. 3, 139-153 (1995)

17. del Pino, M., Felmer, P.: Local mountain passes for semilinear elliptic problems in unbounded domains. Calc. Var. Partial Differ. Equ. 4, 121-137 (1996)

18. do Ó, J.M.: $N$-Laplacian equations in $\mathbb{R}^{N}$ with critical growth. Abstr. Appl. Anal. 2, 301-315 (1997)

19. do Ó, J.M., de Medeiros, E., Severo, U.: A nonhomogeneous elliptic problem involving critical growth in dimension two. J. Math. Anal. Appl. 345, 286-304 (2008)

20. do Ó, J.M., Souto, M.A.S.: On a class of nonlinear Schrödinger equations in $\mathbb{R}^{N}$ involving critical growth. J. Differ. Equ. 174, 289-311 (2001)

21. do Ó, J.M., Sani, F., Zhang, J.: Stationary nonlinear Schrödinger equations in $\mathbb{R}^{N}$ with potentials vanishing at infinity. Ann. Mat. Pura Appl. 196, 363-393 (2017)

22. Fei, M., Yin, H.: Bound states of 2-D nonlinear Schrödinger equations with potentials tending to zero at infinity. SIAM J. Math. Anal. 45, 2299-2331 (2013)

23. Gilbarg, D., Trudinger, N.S.: Elliptic Partial Differential Equations of Second Order. Reprint of the 1998 Edition. Classics in Mathematics. Springer, Berlin (2001)

24. Jeanjean, L., Tanaka, K.: A remark on least energy solutions in $\mathbb{R}^{N}$. Proc. Am. Math. Soc. 131, 2399-2408 (2003)

25. Li, S.J., Santos, C.A., Yang, M.B.: Existence of semiclassical states for a quasilinear Schrödinger equation on RN with exponential critical growth. Acta Math. Sin. (Engl. Ser.) 32, 1279-1296 (2016)

26. Liu, C., Wang, Z., Zhou, H.S.: Asymptotically linear Schrödinger equation with potential vanishing at infinity. J. Differ. Equ. 245, 201-222 (2008)

27. Moroz, V., Van Schaftingen, J.: Semiclassical stationary states for nonlinear Schrödinger equations with fast decaying potentials. Calc. Var. Partial Differ. Equ. 37, 1-27 (2010)

28. Moser, J.: A sharp form of an inequality by N. Trudinger. Indiana Univ. Math. J. 20, 1077-1092 (1970/71)

29. Ni, W.-M., Takagi, I.: On the shape of least-energy solutions to a semilinear Neumann problem. Commun. Pure Appl. Math. 44, 819-851 (1991)

30. Ogawa, T.: A proof of Trudinger's inequality and its application to nonlinear Schrödinger equations. Nonlinear Anal. 14, 765-769 (1990)

31. Ogawa, T., Ozawa, T.: Trudinger type inequalities and uniqueness of weak solutions for the nonlinear Schrödinger mixed problem. J. Math. Anal. Appl. 155, 531-540 (1991)

32. Opic, B., Kufner, A.: Hardy-Type Inequalities. Pitman Research Notes in Mathematics Series, vol. 219. Longman Scientific and Technical, Harlow (1990)

33. Ozawa, T.: On critical cases of Sobolev's inequalities. J. Funct. Anal. 127, 259-269 (1995)

34. Rabinowitz, P.H.: A note on a semilinear elliptic equation on $\mathbb{R}^{N}$. Nonlinear analysis. Sc. Norm. Super. di Pisa Quaderni, Scuola Norm. Sup., Pisa, 307-317 (1991)

35. Ruf, B.: A sharp Trudinger-Moser type inequality for unbounded domains in $\mathbb{R}^{2}$. J. Funct. Anal. 219 , 340-367 (2005)

36. Tao, B., Liu, Z., Yang, M.: Semiclassical ground state solutions for a Schrödinger equation in $\mathbb{R}^{2}$ with critical exponential growth. Math. Nachr. 289, 727-747 (2016)

37. Yang, Y., Zhu, X.: A new proof of subcritical Trudinger-Moser inequalities on the whole Euclidean space. J. Partial Differ. Equ. 26, 300-304 (2013) 
38. Zhang, J., do Ó, J.M.: Standing waves for nonlinear Schrödinger equations involving critical growth of Trudinger-Moser type. Z. Angew. Math. Phys. 66, 3049-3060 (2015)

Publisher's Note Springer Nature remains neutral with regard to jurisdictional claims in published maps and institutional affiliations. 